\title{
Bone marrow niche ATP levels determine leukemia- initiating cell activity via P2X7 in leukemic models
}

\author{
Xiaoxiao He, Jiangbo Wan, ${ }^{2}$ Xiaona Yang, ${ }^{3}$ Xiuze Zhang, ${ }^{4}$ Dan Huang, ${ }^{1}$ Xie Li, ${ }^{4}$ Yejun Zou, ${ }^{4}$ Chiqi Chen, ${ }^{1}$ Zhuo Yu, ${ }^{1}$ Li Xie, ${ }^{1}$ \\ Yaping Zhang, ${ }^{1}$ Ligen Liu, ${ }^{1}$ Shangang Li, ${ }^{5}$ Yuzheng Zhao, ${ }^{4}$ Hongfang Shao, ${ }^{6} \mathrm{Ye} \mathrm{Yu},{ }^{3}$ and Junke Zheng,
}

\begin{abstract}
'Hongqiao International Institute of Medicine, Shanghai Tongren Hospital, Key Laboratory of Cell Differentiation and Apoptosis of Chinese Ministry of Education, Faculty of Basic Medicine, Shanghai Jiao Tong University School of Medicine, Shanghai, China. '2Department of Hematology, Xinhua Hospital, Affiliated to Shanghai liao Tong University School of Medicine, Shanghai, China. ${ }^{3}$ School of Basic Medicine and Clinical Pharmacy, China Pharmaceutical University, Nanjing, China. ${ }^{4}$ Optogenetics \& Synthetic Biology Interdisciplinary Research Center, State Key Laboratory of Bioreactor Engineering, Research Unit of Chinese Academy of Medical Sciences, School of Pharmacy, East China University of Science and Technology, Shanghai, China. ${ }^{5}$ Yunnan Key Laboratory of Primate Biomedicine Research, Institute of Primate Translational Medicine, Kunming University of Science and Technology, Kunming, China. ${ }^{6}$ Center of Reproductive Medicine, Shanghai Sixth People's Hospital, Shanghai, China. ${ }^{~ S h a n g h a i ~ K e y ~}$ Laboratory of Reproductive Medicine, Shanghai Jiao Tong University School of Medicine, Shanghai, China.
\end{abstract}

\begin{abstract}
How particular bone marrow niche factors contribute to the leukemogenic activities of leukemia-initiating cells (LICs) remains largely unknown. Here, we showed that ATP levels were markedly increased in the bone marrow niches of mice with acute myeloid leukemia (AML), and LICs preferentially localized to the endosteal niche with relatively high ATP levels, as indicated by a sensitive ATP indicator. ATP could efficiently induce the influx of ions into LICs in an MLL-AF9-induced murine AML model via the ligand-gated ion channel P2X7. P2x7 deletion led to notably impaired homing and self-renewal capacities of LICs and contributed to an approximately 5-fold decrease in the number of functional LICs but had no effect on normal hematopoiesis. ATP/P2X7 signaling enhanced the calcium flux-mediated phosphorylation of CREB, which further transactivated phosphoglycerate dehydrogenase (Phgdh) expression to maintain serine metabolism and LIC fates. P2X7 knockdown resulted in a markedly extended survival of recipients transplanted with either human AML cell lines or primary leukemia cells. Blockade of ATP/P2X7 signaling could efficiently inhibit leukemogenesis. Here, we provide a perspective for understanding how ATP/P2X7 signaling sustains LIC activities, which may benefit the development of specific strategies for targeting LICs or other types of cancer stem cells.
\end{abstract}

\section{Introduction}

Acute myeloid leukemia (AML) is a severe hematological malignant disease that is characterized by the clonal expansion of hematopoietic stem/progenitor cells (1). AML is the major type of acute leukemia in adults and has high mortality (approximately 65\%) and relapse (approximately 50\%) rates (2). It has been reported that there is a small population of leukemia-initiating cells (LICs) that can self-renew and give rise to all the bulk leukemia cell progenies (3). LICs are also resistant to chemotherapy, which leads to the relapse of AML (4). Nevertheless, the mechanism by which LICs maintain their leukemogenic activities remains largely unknown.

Increasing evidence has suggested that bone marrow (BM) niche factors play important roles in leukemogenesis or resistance to conventional chemotherapy or radiotherapy treatments $(5,6)$. Although many different niche factors, such as CCL3, GDF1, IL-6, selectins, and hyaluronic acid, have been shown to participate in sustaining the LIC pool (7-11), the details of the niche components and regulatory networks involved in this process are still poorly

Authorship note: $\mathrm{XH}$ and JW contributed equally to this work.

Conflict of interest: The authors have declared that no conflict of interest exists.

Copyright: @ 2021, American Society for Clinical Investigation.

Submitted: May 15, 2020; Accepted: December 9, 2020; Published: February 15, 2021.

Reference information: J Clin Invest. 2021;131(4):e140242.

https://doi.org/10.1172/JCl140242. understood. We recently reported that several lipid metabolismrelated regulators, including ANGPTL2 and APOE, can bind to certain immune inhibitory receptors, including LILRB2 and LILRB4, to promote leukemogenesis by enhancing self-renewal and migration or by suppressing $\mathrm{T}$ cell responses $(12,13)$. We further showed that the glycolytic preference of AML LICs determines their homing and localization to the endosteal niche rather than the vascular niche (14). These findings indicate that there may be certain metabolic niches that tightly control the fates and activities of LICs, although the detailed mechanisms require further investigation.

Nucleotides, mainly ATP, UTP, ADP, and UDP, have been reported to be abundantly present in the extracellular niche/ microenvironment; for example, it has been found that ATP levels in the microenvironment of solid cancers are in the range of 100-500 micromoles per liter, which is much higher than that in normal tissues $(10-100$ nanomoles per liter) $(15,16)$. These nucleotides may play important roles in cell-to-cell communication and signal transduction during many physiological and pathological processes by binding to different types of purinergic receptors (P2Rs) (17-19). The P2R family can further be subdivided into 2 subgroups, namely, the ligand-gated ion channels (P2X, which only bind ATP) and G protein-coupled membrane receptors (P2Y, which bind ATP, ADP, UTP, and UDP) $(20,21)$. Upon ATP stimulation, the P2X family members (P2X1-P2X7) mainly act as membrane channels to mediate the influx of ions into cells, including 
cells of the hematopoietic system, to regulate cell proliferation, differentiation, migration, and death (22-24). For example, ATP enhances the proliferation of hematopoietic stem cells (HSCs) and maintains the size of the hematopoietic progenitor population, especially during chronic inflammation, mainly through P2X1 and $\mathrm{P} 2 \mathrm{X} 4$. ATP, in combination with other cytokines, including IL-3 and GM-CSF, can also induce myeloid differentiation (25). Both ATP and UTP can promote the proliferation of human HSCs in vitro or in vivo (19). Interestingly, extracellular UTP and, to a lesser extent, ATP, are required for the regulation of HSC motility and homing (26). However, the detailed functions of the different P2X members in the regulation of HSC stemness or malignant transformation remain largely unknown.

Several studies have shown that extracellular ATP has a suppressive effect in cancers, including hematologic malignancies $(16,27)$. For example, ATP can inhibit the growth and enhance the differentiation of human HL-60 or NB4 AML cells $(28,29)$. However, studies have also shown that P2Xs, especially the P2X7 subtype, are expressed in many types of cancer, including chronic B cell lymphocytic leukemia (B-CLL), prostate cancer, breast cancer, neuroblastoma, and epithelial cancers, and enhance the progression and drug resistance of these cancers (30, 31). P2X7 can also inhibit the antitumor effect of $\mathrm{CD}^{+} \mathrm{T}$ cells to enhance tumorigenesis and metastasis $(32,33)$. P2X7 or other P2X members (including P2X1 and P2X4) have also been found to be highly expressed in myeloid disorders, such as myelodysplastic syndrome, chronic myeloid leukemia, and AML (34-36). Recent evidence further indicates that ectopic $\mathrm{P} 2 \mathrm{X} 7$ overexpression may enhance AML development but impair normal hematopoiesis $(37,38)$. In light of these seemingly contradictory functions of $\mathrm{P} 2 \mathrm{Xs}$, it is essential to use genetic tools, such as P2X-knockout mice, to delineate how ATP levels are changed or distributed in different BM niches and how ATP/P2X-mediated signaling affects leukemogenesis.

Compared with the other P2X subtypes, P2X7 is unique because it has the longest C-terminal intracellular domain, and forms a pore that is permeable to large cations upon stimulation. After extracellular ATP binds to P2X7, the ion channel opens and allows the influx of calcium and the efflux of potassium (39). During repeated or prolonged ATP stimulation, P2X7 continues to open and form pores that allow larger molecules to enter cells (40). P2X7 can also indirectly trigger voltage-gated calcium channels to regulate calcium-dependent signal transduction pathways (41), and P2X7 plays an important role in a variety of physiological and pathological processes $(15,32)$. Preclinical studies have also shown that P2X7 antagonists can potentially be used for the treatment of many disorders, especially inflammatory disorders and cancers $(42,43)$. However, the mechanism by which ATP/P2X7 signaling regulates the leukemogenic activities of LICs in BM niches and its potential therapeutic role in leukemia treatment are still not fully understood.

In this study, we demonstrated that ATP levels are markedly increased in the BM niches of AML mice and that LICs tend to be localized to the endosteal niche, which has high ATP levels, by using a genetically encoded ATP sensor (iATPSnFR). We generated $P 2 x 7$-knockout mice and provide several lines of evidence showing that P2X7 is important for the maintenance of LIC leuke- mogenic activities in an MLL-AF9-induced murine model of AML but is not required for the maintenance of HSC stemness. Extracellular ATP efficiently induces the P2X7-mediated influx of ions, which leads to enhanced calcium signaling-mediated direct transactivation of phosphoglycerate dehydrogenase ( $P h g d h)$ expression and maintenance of normal serine metabolism to control LIC homing and self-renewal abilities. P2X7 is also required for the proliferation of human AML cell lines and primary LICs. Treatment with P2X7 antagonists or depletion of serine can effectively inhibit the proliferation of AML cells both in vitro and in vivo.

\section{Results}

The endosteal niche has a much higher ATP level than the vascular niche. To determine how the niche component ATP affects LIC fate, we first measured the ATP concentration in the BM fluid from leukemic and control mice transplanted with BM cells expressing an empty vector 4 months after injection and found that the ATP level in the leukemic mice was approximately 950-fold higher than that in the control mice ( 231 vs. $0.24 \mu \mathrm{M}$, Figure $1 \mathrm{~A}$ ). To precisely evaluate the dynamic changes in the ATP levels in the BM niches, we took advantage of genetically encoded fluorescent sensor iATPSnFR for cell surface ATP detection $(44,45)$ and generated a ratiometric ATP sensor by fusing the red fluorescent protein mCherry to the N-terminus of iATPSnFR (mCherry-iATPSnFR, Figure 1B) and a nonresponsive control sensor (mCherrycpSFGFP, NC). To test whether iATPSnFR can be used to sensitively measure extracellular ATP levels in vitro, we firstly transfected iATPSnFR and the control sensor into a human AML cell line, U937 cells, and examined the changes in iATPSnFR fluorescence at excitation wavelengths of $488 \mathrm{~nm}$ and $561 \mathrm{~nm}$. As shown in Figure 1C, cell surface mCherry-iATPSnFR displayed clear changes in green fluorescence (to measure the ATP level) in the presence of ATP, whereas the red fluorescence (to normalize the iATPSnFR sensor protein level in individual cells) of mCherry did not. ATP stimulation in vitro led to an approximately 2-fold increase in the fluorescence ratio $(488 \mathrm{~nm} / 561 \mathrm{~nm})$ of iATPSnFR but did not increase the fluorescence ratio of the control sensor mCherry-cpSFGFP (Figure 1, C and D, and Supplemental Figure 1A; supplemental material available online with this article; https://oi.org/10.1172/JCI140242DS1). Currently, the vascular niche and endosteal niche are usually defined as the regions where the leukemia cells directly contact or are within one-cell distance from the endothelial cells and osteoblasts in the BM, respectively. To test whether iATPSnFR can indicate the distribution of ATP in different BM niches, we transplanted iATPSnFR-U937 cells and NC-U937 cells into NOD-SCID mice and found that the fluorescence ratio $(488 \mathrm{~nm} / 561 \mathrm{~nm})$ of the iATPSnFR-U937 cells residing near the endosteal niche was much higher than that of the cells residing near the vascular niche (Figure 1, E-F). Moreover, the closer the cells were to the endosteal niche, the higher the iATPSnFR fluorescence ratio was (Figure 1G). In contrast, in the mice transplanted with the NC-U937 cells, no significant difference was observed in the fluorescence ratio of the cells located near the endosteal niche and that of the cells located near the vascular niche (Supplemental Figure 1, B-D).

To demonstrate whether the ATP level as determined by a human AML cell line was applicable to a primary murine AML mod- 
A

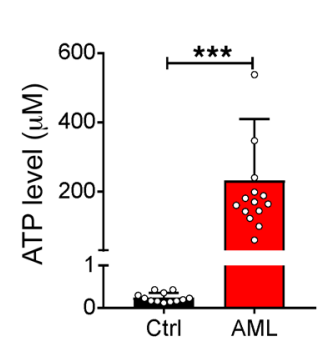

B

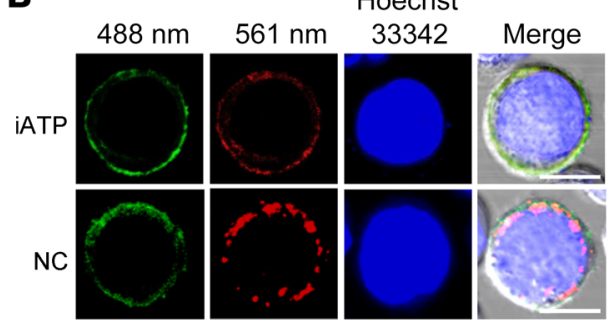

C

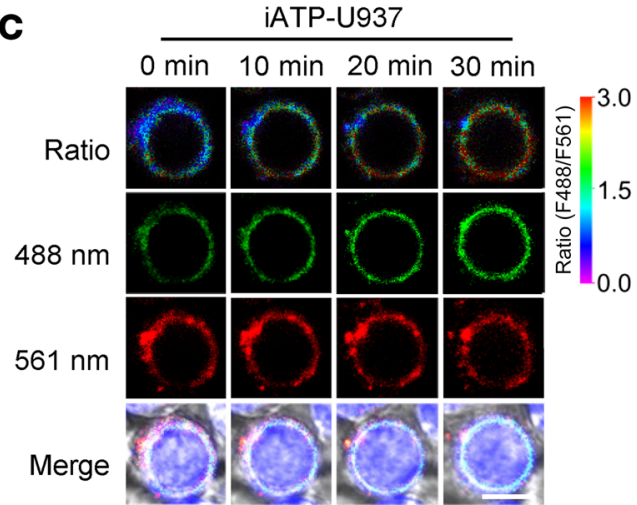

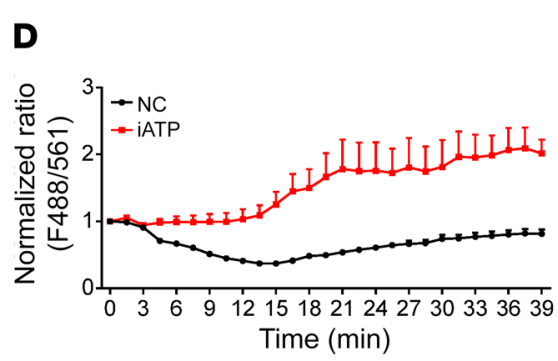

iATP-U937

E Ratio (488/561)/
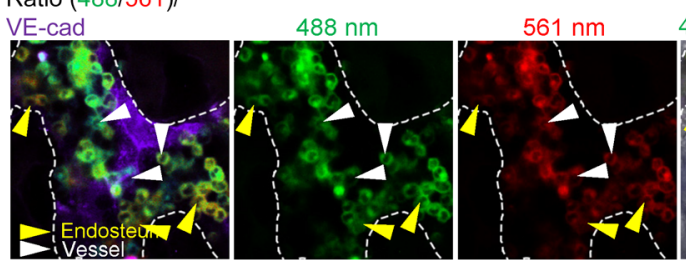

488/561/NE-cad/DIC

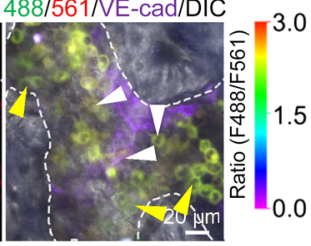

$\mathbf{F}$

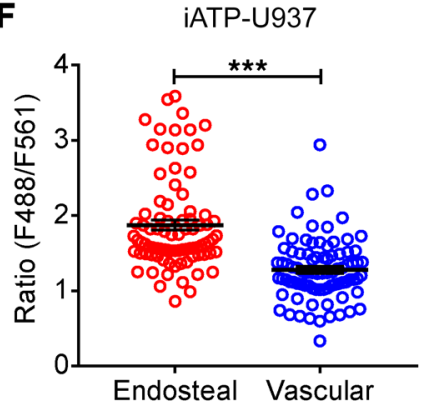

G

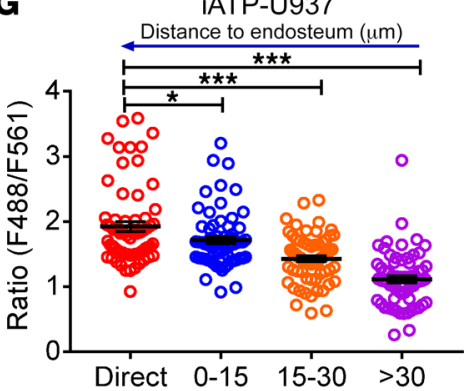

Figure 1. The endosteal niche has a much higher ATP level than the vascular niche. (A) Biochemical analysis of ATP levels in BM fluid of control and leukemic mice (Ctrl, $n=12$; AML, $n=15$ ). ${ }^{* * *} P<0.001$ by Student's $t$ test. (B) Representative images of U937 cells infected with the ATP sensor (iATPSnFR) or the control sensor (mCherry-cpSFCFP, NC). Nuclei were stained with Hoechst 33342. (C) Representative images of the ratio of iATPSnFR fluorescence (F488/F561 nm) at excitation wavelengths of $488 \mathrm{~nm}$ and $561 \mathrm{~nm}$ in iATPSnFR-U937 cells at the indicated time points upon ATP treatment. Scale bars (B and C): $10 \mu \mathrm{m}$. (D) Quantification of the fluorescence ratio (F488/F561 nm) of iATPSnFR and the control sensor in iATPSnFR-U937 cells upon ATP treatment ( $n=41$ cells/group). (E) Representative images of the localization of iATPSnFR-U937 cells in the endosteal niche (dashed lines) and vascular niche (labeled with anti-VE-cadherin) and their ratios of iATPSnFR fluorescence (488/561 nm) are shown. (F) Quantification of the iATPSnFR fluorescence ratio in iATPSnFR-U937 cells in the endosteal and the vascular niche in panel E ( $n=$ approximately 85 cells/group from 3 biological replicates). (G) Quantification of the iATPSnFR fluorescence ratio in iATPSnFR-U937 cells and its relationship to the cells' distance from the endosteum in panel $\mathbf{E}$ ( $n=$ approximately 65 cells/group from 3 biological replicates). ${ }^{*} P<0.05 ;{ }^{* *} P<0.001$ by 1 -way ANOVA with Tukey's multiple-comparison test.

el, we overexpressed iATPSnFR or the control sensor in an MLLAF9-induced murine AML model to determine whether it was also able to efficiently reflect the ATP levels in different BM niches. We demonstrated that exogenous ATP could also increase the fluorescence ratio of iATPSnFR-AML cells in vitro by up to 2-fold (Supplemental Figure 1, E-G); however, exogenous ATP did not increase the fluorescence ratio of NC-AML cells. In vivo imaging analysis also revealed that the fluorescence ratio of the iATPSnFR-AML cells residing near the endosteal niche was 1.5 -fold higher than that of the iATPSnFR-AML cells residing near the vascular niche in the BM of C57BL/6 leukemic mice (Supplemental Figure 1, H-I). Similar to our observations in the iATPSnFR-U937 cells, the closer the cells were to the endosteal niche, the higher the iATPSnFR fluorescence ratio was (Supplemental Figure 1J); however, this difference was not observed in the NC-MLL-AF9 leukemic mouse model (Supplemental Figure 1, K-M). These results further confirmed that ATP levels have different distribution patterns in different BM niches. Consistently, Col2.3 $3^{+}$osteoblasts seemed to produce more ATP than either $\mathrm{CD} 1^{+} \mathrm{CD} 45^{-} \mathrm{Ter}^{-119}{ }^{-}$endothelial cells or CD31-CD45- ${ }^{-}$Ter119- ${ }^{-} \mathrm{Lepr}^{+}$ mesenchymal stem cells (MSCs), as determined by an in vitro assay. Osteoblasts also had the highest mRNA levels of pannexin 1 (Panx1) and connexin $43(C x 43)$, which are 2 key regulators $(46,47)$ that enhance ATP secretion into the extracellular microenvironment (Supplemental Figure 1, N-O). As we have previously shown that AML LICs prefer to localize to the endosteal niche rather than the vascular niche (14), it is possible that the high ATP levels in the endosteal niche may contribute to the retention of LICs in specific BM niches and to the maintenance of their leukemogenic activities. 
A

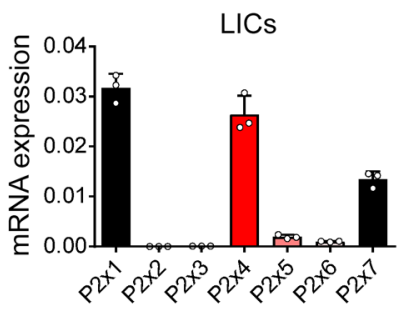

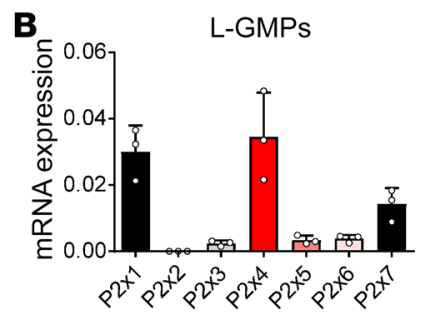

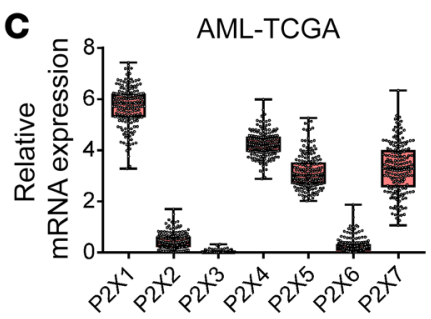

D

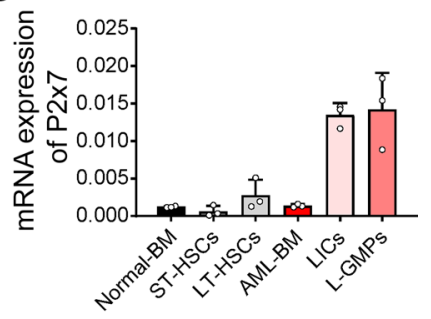

$\mathbf{E}$ Mouse LICs $1 \mathrm{mM} \mathrm{ATP}$ in $\mathrm{Ca}^{2+}{ }_{-}$free $\quad 5 \mu \mathrm{M} \mathrm{A-740003}$

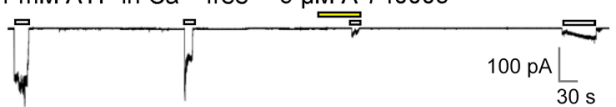

$\mathbf{F}$

CRISPR-Cas9 for P2x7-KO mice WT GgGgtgaCGARGTTAgGaCACAGCATCTTTGAC

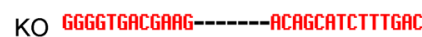
$\mathrm{KO}: 7 \mathrm{bp}$ deletion

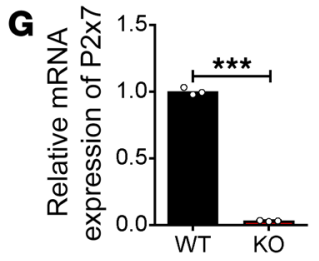

H

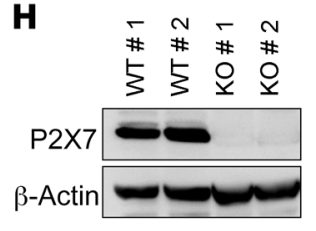

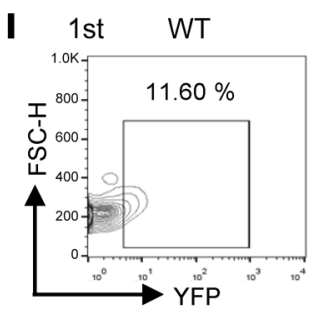

M

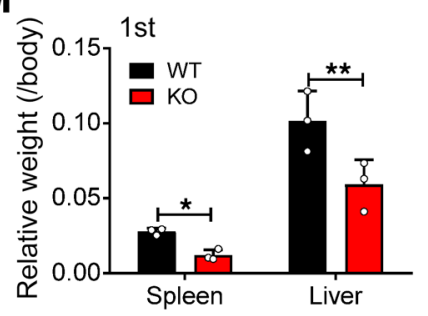

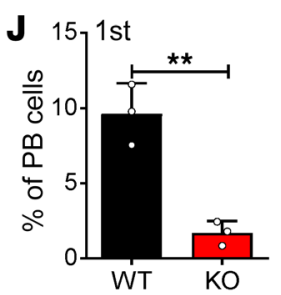

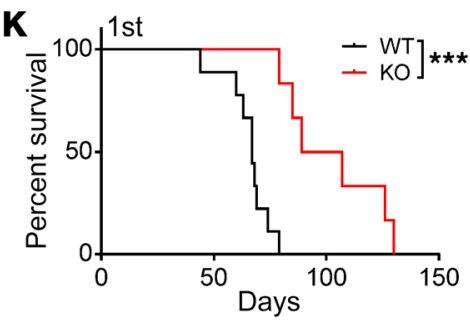

L 1st

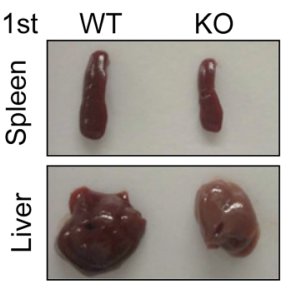

O

$\mathbf{N}$

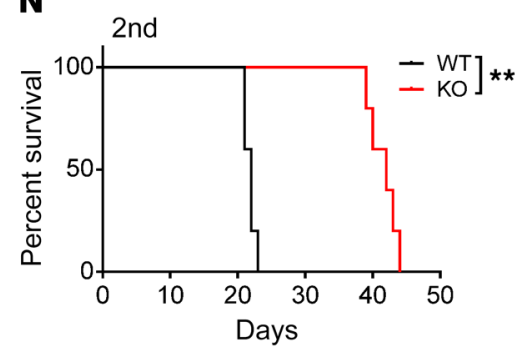

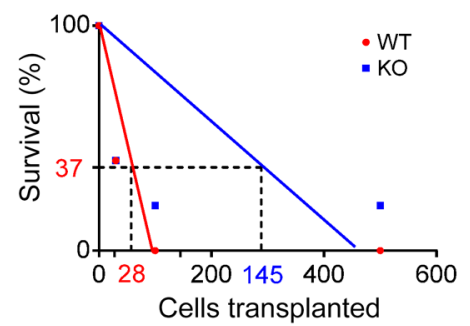

Figure 2. P2X7 is highly expressed in LICs and promotes AML development. ( $\mathbf{A}$ and $\mathbf{B}$ ) mRNA levels of $P 2 \times 1-P 2 \times 7$ were determined in the immunophenotypic Mac-1+c-Kit' LICs (A) or Lin-Sca-1-c-Kit+CD34+CD16/32- L-GMPs (B) by quantitative RT-PCR $(n=3)$. (C) mRNA levels of P2Xs were analyzed in cells of AML patients from TCCA database $(n=179)$. (D) mRNA levels of $P 2 x 7$ in normal mouse bone marrow cells (Normal-BM), Lin ${ }^{-}$Sca- $1^{+} \mathrm{C}-\mathrm{Kit}^{+} \mathrm{CD} 34^{-} \mathrm{Flk2} 2^{-}$long-term HSCs (LT-HSCs), Lin-Sca-1+c-Kit+CD34+Flk2+ short-term HSCs (ST-HSCs), YFP+ leukemia cells (AML-BM), Mac-1+c-Kit' LICs, and Lin-Sca-1-c-Kit+CD34+CD16/32L-CMPs were measured by quantitative RT-PCR $(n=3)$. (E) P2X7-mediated influx of ions in murine Mac- $1^{+} \mathrm{C}-\mathrm{Kit}^{+}$LICs cells was measured upon sequential treatments with extracellular ATP and P2X7 antagonist A-740003 by whole-cell patch-clamp recording $(n=3)$. (F) Nucleotide base sequences deleted in the $P 2 \times 7$-knockout ( $P 2 \times 7-K O$ or $P 2 \times 7$-null) mouse using the CRISPR-Cas9 strategy. (G) mRNA level of $P 2 \times 7$ in WT and $P 2 \times 7-K O$ BM cells was measured by quantitative RT-PCR $(n=3)$. ${ }^{* *} P<0.001$ by Student's $t$ test. (H) P2X7 protein levels in WT and P2x7-KO BM cells were measured by Western blot. (I) Representative flow cytometric analysis of leukemia cells (YFP+) in the peripheral blood upon primary transplantation. (J) Quantification of data in panel I ( $n$ $=5$ ). PB, peripheral blood. ${ }^{* *} P<0.01$ by Student's $t$ test. (K) The overall survival of the recipient mice transplanted with WT or P2x7-KO MLL-AF9+ BM cells upon primary transplantation $(n=5)$. ${ }^{* *} P<0.001$ by log-rank test. (L) Representative images of the size of spleens and livers of recipients upon primary transplantation. (M) Quantification of data in panel $\mathbf{L}(n=4) .{ }^{*} P<0.05$, ${ }^{* *} P<0.01$ by 2-way ANOVA with Sidak's multiple-comparison test. (N) The overall survival of recipient mice transplanted with WT or $P 2 \times 7-K O$ MLL-AF9+ BM cells upon secondary transplantation $(n=5)$. ${ }^{* * *} P<0.001$ by log-rank test. $(\mathbf{0})$ Limiting dilution assays for the frequency of the functional LICs of WT and P2x7-KO BM cells. Experiments were repeated independently 3 times.

$P 2 X 7$ is highly expressed in LICs and promotes AML development. To understand whether the extracellular ATP levels are associated with LIC activities in the endosteal niche, we first examined the mRNA expression levels of all the P2X ATP-gated ion channels (P2X1-P2X7) in the LICs of mice with MLL-AF9-induced AML; in these mice, the AML cells only expressed myeloid cell markers (Mac-1 and Gr-1) but not lymphoid cell markers (CD3 and B220), as previously described (48). Interestingly, $P 2 x 1,-4$, and -7 were abundant in both the immunophenotypic Mac- $1^{+} \mathrm{c}-\mathrm{Kit}^{+} \mathrm{LICs}$ and
Lin-Sca- $1^{+} \mathrm{c}-\mathrm{Kit}^{+} \mathrm{CD} 34^{+} \mathrm{CD} 16 / \mathrm{CD} 32^{+}$L-GMP cells (a population more highly enriched in LICs; Figure 2, A and B). Consistent with the expression patterns in the mouse AML LICs, several P2X members, including $P 2 X 1, P 2 X 4, P 2 X 5$, and $P 2 X 7$, were highly expressed in the human AML cells from The Cancer Genome Atlas (TCGA) database (Figure 2C). Because P2X7 has been reported to exhibit completely different patterns of ion flux and formation of cytolytic pores that are permeable to large cations upon repeated ATP stimulation $(40,49)$, we next decided to exam- 
ine the function of P2X7 during leukemogenesis. We first examined the mRNA levels of $P 2 x 7$ in both normal hematopoietic cells and leukemia cells. Interestingly, the immunophenotypic Mac- $1^{+}$ c-Kit ${ }^{+}$LICs and Lin ${ }^{-} \mathrm{Sca}-1^{+} \mathrm{c}-\mathrm{Kit}^{+} \mathrm{CD} 34^{+} \mathrm{CD} 16 / \mathrm{CD} 32^{+} \mathrm{L}-\mathrm{GMP}$ cells expressed high levels of $P 2 x 7$, and the expression of $P 2 x 7$ in these cells was approximately 5 - to 10 -fold higher than that in the bulk AML cells and long-term HSCs (LT-HSCs) (Figure 2D). Moreover, the P2X7-mediated ion flux in the murine AML LICs could be efficiently induced by stimulation with extracellular ATP and blocked by A-740003, a potent and selective P2X7 inhibitor, as determined by whole-cell patch-clamp electrophysiology recording (Figure $2 \mathrm{E}$ ); these results indicated that the $\mathrm{ATP} / \mathrm{P} 2 \mathrm{X} 7$-mediated signal is important for the leukemogenic activities of AML-LICs.

To address the potential functions of $\mathrm{P} 2 \mathrm{X} 7$ in leukemogenesis, $P 2 x 7$ was knocked down in MLL-AF9 ${ }^{+}$murine AML cells by shRNA (Supplemental Figure 2, A and B), and these cells were transplanted into recipient mice. AML development was much slower in the mice transplanted with the $P 2 x 7$-knockdown cells than in the control mice, as evidenced by the decreased frequency of leukemia cells in the peripheral blood (Supplemental Figure 2, C and D) and the reduced infiltration in the livers and spleens of the transplant recipients (Supplemental Figure 2, E and F). More importantly, the overall survival of the leukemic mice transplanted with the P2x7-knockdown cells was markedly extended compared with that of the control mice upon either primary (45 or 51 vs. 26 days, Supplemental Figure 2G) or secondary transplantation (29 or 32 vs. 25 days, Supplemental Figure $2 \mathrm{H}$ ), indicating that $\mathrm{P} 2 \mathrm{X} 7$ may be required for the self-renewal capacity of LICs.

To further confirm the function of $\mathrm{P} 2 \mathrm{X} 7$, we generated $P 2 x 7$-knockout (P2x7-KO) mice (Figure $2 \mathrm{~F}$ ) using the CRISPRCas9 strategy and demonstrated that almost no detectable $P 2 x 7$ mRNA or protein levels (Figure 2, G and $\mathrm{H}$ ) were observed in the total BM cells of the P2x7-KO mice. We then established an MLLAF9-induced AML model with wild-type (WT) and KO mice and revealed that leukemia development was much slower in the mice transplanted with P2x7-null AML cells than in the control mice, as evidenced by the decreased frequency of $\mathrm{YFP}^{+}$leukemia cells in the peripheral blood (Figure 2, I and J), the reduced percentages of blast cells in both the peripheral blood and BM (Supplemental Figure 2, I-L), the dramatically increased overall survival (67 vs. 98 days, Figure 2K), and the reduced infiltration in the livers and spleens of the transplant recipients after primary transplantation (Figure 2, L and M, and Supplemental Figure $2 \mathrm{M})$. The survival of the mice transplanted with primary $P 2 x 7-$ null cells was significantly prolonged compared with that of the mice transplanted with WT cells after secondary transplantation (22 vs. 42 days, Figure 2N). Limiting dilution assays with the $\mathrm{YFP}^{+} \mathrm{AML}$ cells from the primary transplant recipients revealed that the frequency of functional LICs was 1 in 28, which was approximately 5.2 -fold higher than that in the $P 2 x 7$-null controls (1 in 145, Supplemental Table 1 and Figure 2O). Taken together, these results clearly suggest that ATP/P2X7-mediated pathways enhance leukemogenesis and may be an ideal target in LICs. In contrast, ATP/P2X7 signaling seemed to have no effects on the normal repopulation of HSCs (Supplemental Figure 2, N-P), suggesting that ATP/P2X7 signaling may be an ideal target for LIC-targeted therapy.
ATP/P2X7 signaling promotes the homing and self-renewal of LICs. To determine how ATP/P2X7 signaling affects the fate of LICs, we first analyzed the migratory abilities of $P 2 x 7$-null AML bulk cells and LICs by using a Transwell assay. As shown in Figure $3 \mathrm{~A}$, the percentages of migrated $P 2 x 7$-null bulk leukemia cells and LICs were much lower after 4 hours of culture. Moreover, we also performed a homing assay to further confirm the change in the migratory ability of the $P 2 x 7$-null AML cells and found that there were approximately $50 \%-60 \%$ fewer cells that homed to the BM in the recipient mice transplanted with $P 2 x 7$-null leukemia cells than in the recipient mice transplanted with WT cells (Figure 3B and Supplemental Figure 3A). Because the ATP level was much higher in the endosteal niches (Figure 1, E and F, and Supplemental Figure $1, \mathrm{H}$ and I), we speculated that the deletion of $P 2 x 7$ may have affected the localization of the LICs. We performed ex vivo imaging analysis of the cranium of the leukemic mice and found that a much higher percentage of WT AML cells resided in the endosteal niche than P2x7-null AML cells (35.71\% vs. $18.60 \%$; Supplemental Figure 3, B and C). Immunostaining analysis further showed that approximately $47.80 \%$ of the LICs in the WT leukemic mice were mainly localized to the endosteal niche, while only $19.56 \%$ of these cells resided near the vascular niche; however, in the $P 2 x 7$-null leukemic recipients, approximately $17.90 \%$ and $49.50 \%$ of the LICs were localized to the endosteal niche and vascular niche, respectively (Figure 3, C and D). These results indicated that $\mathrm{ATP} / \mathrm{P} 2 \mathrm{X} 7$ signaling may be important for the retention of LICs in the endosteal niche.

We then further evaluated whether ATP/P2X7 signaling is required for the commitment of LICs to other fates by examining the LIC frequency after serial transplantation. Interestingly, we found that the frequency of the immunophenotypic Mac- $1^{+} \mathrm{c}-\mathrm{Kit}^{+}$ P2x7-null LICs was reduced to $70 \%$ or $50 \%$ of the frequency of the WT LICs upon primary (Figure 3E and Supplemental Figure 3D) or secondary transplantation (Figure $3 \mathrm{~F}$ and Supplemental Figure $3 \mathrm{E}$ ). Moreover, the frequencies of the $\mathrm{Lin}^{-} \mathrm{CD} 127^{-} \mathrm{Sca}-{ }^{-} \mathrm{C}-\mathrm{Kit}^{+}$ $\mathrm{CD} 34^{+} \mathrm{CD} 16 / 32^{+} \mathrm{P} 2 x 7$-null L-GMP cells, which is a population that has been indicated to be more highly enriched in immunophenotypic LICs (50), were also much lower than those of WT control cells (Supplemental Figure 3, F-H). An in vitro surrogate functional analysis with methylcellulose medium further revealed a dramatic decrease in the colony size, colony number, and derived cell number of the $P 2 x 7$-null AML cells during both primary plating and secondary plating (Figure 3, G and H, and Supplemental Figure 3I). We also used an alternative way to exclude the homing effect by using intratibial injection with AML cells. Interestingly, the leukemia development was still much slower in the recipient mice receiving P2x7-null AML cells than that of the WT control, as evidenced by the reduced leukemia cell frequency in peripheral blood and extended overall survival (Figure 3, I and J). These results suggest that both homing and proliferation defects may contribute to the phenotypes in $P 2 x 7-\mathrm{KO}$ leukemic mice. In addition, there was no significant difference in apoptosis (Supplemental Figure 3, J and K) or differentiation (Supplemental Figure 3L), as measured by annexin V/7-AAD staining or Mac-1/Gr-1 staining (Gr-1 expression level represents the degree of differentiation), respectively. Consistently, the LIC frequency was substantially reduced in the mice transplanted with $P 2 x 7-$ knockdown cells 
A

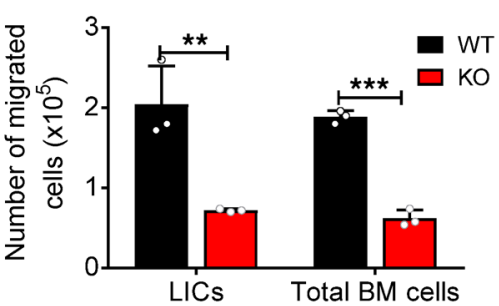

D
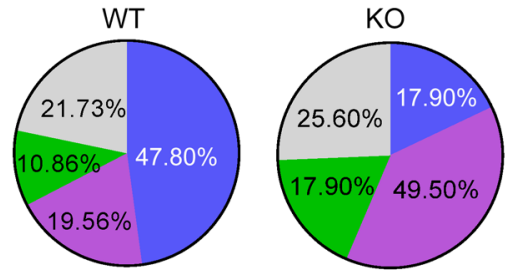

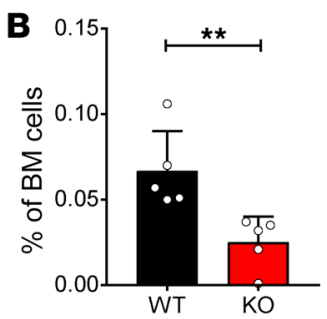

C
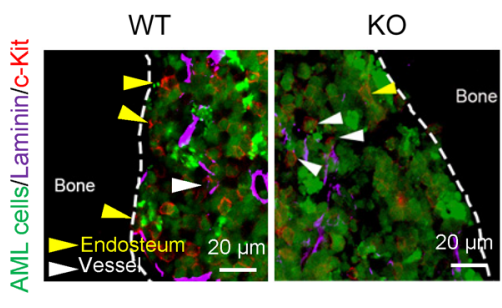

Attach to endosteum Attach to vessel

Attach to both

Other
G

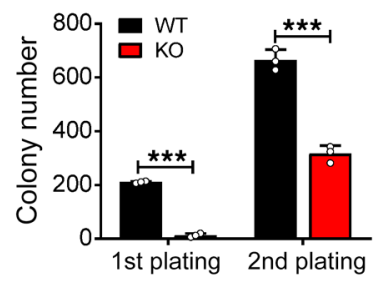

H

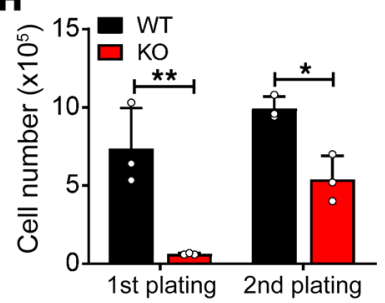

E

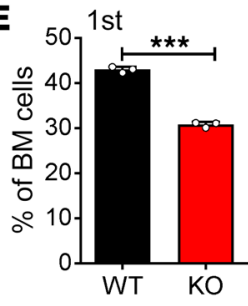

$\mathbf{F}$

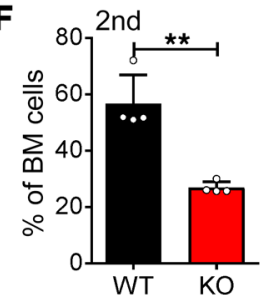

I

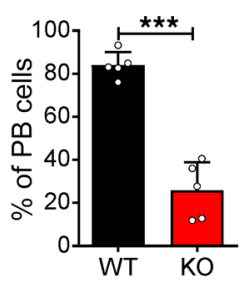

J

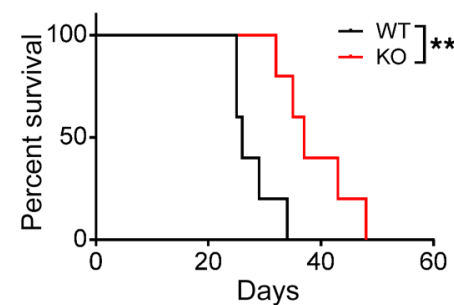

Figure 3. ATP/P2X7 signaling maintains the homing and self-renewal abilities of LICs. (A) Quantification of the migratory abilities of WT and $P 2 \times 7-K O$ Mac- $1^{+}$C-Kit ${ }^{+}$LICs or total BM cells evaluated by Transwell assay $(n=3) .{ }^{* *} P<0.01,{ }^{* *} P<0.001$ by 2-way ANOVA with Sidak's multiple-comparison test. (B) The frequency of homed WT and $P 2 \times 7-$ KO leukemia cells (CFSE ${ }^{+}$) in the BM of the recipients 16 hours after transplantation $(n=5)$. ${ }^{* *} P<0.01$ by Student's $t$ test. (C) Representative images of immunofluorescence staining for the localization of LICs (red) in the laminin ${ }^{+}$vascular niche (purple) or the endosteal niche (dashed lines) in the femur of AML mice. AML cells are indicated by GFP positivity (green). Scale bars: $20 \mu \mathrm{m}$. (D) Percentages of $\mathrm{Mac}-1^{+} \mathrm{C}-\mathrm{Kit}{ }^{+} \mathrm{LICs}$ attached to the endosteal niche, the vascular niche, or both in panel $\mathbf{C}$ were calculated ( $n=\sim 50$ cells/group from 4 biological replicates) (E and $\mathbf{F})$ The frequency of WT and P2x7-KO Mac-1+C-Kit ${ }^{+}$LICs of the recipients upon primary $(\mathbf{E})$ and secondary $(\mathbf{F})$ transplantation $(n=3)$. ${ }^{* * *} P<0.001$ by Student's $t$ test. (G and $\mathbf{H}$ ) Colony numbers $(\mathbf{G})$ and derived total cell count $(\mathbf{H})$ of WT and P2x7-KO YFP+ AML cells during the first and second plating were calculated $(n=3) .{ }^{*} P<0.05$, ${ }^{* *} P<0.01,{ }^{* * *} P<0.001$ by 2 -way ANOVA with Sidak's multiple-comparison test. (I) Leukemia cells were transplanted into recipient mice by intratibial injection. Shown is the quantification of the frequency of the leukemia cells (YFP+) in the peripheral blood of recipient mice 3 weeks after transplantation $(n=5) .{ }^{* *} P<0.001$ by Student's $t$ test. (J) Overall survival was determined in the leukemic mice in panel I $(n=5)$. ${ }^{* *} P<0.01$ by log-rank test.

compared with the mice transplanted with the WT cells after serial transplantation (Supplemental Figure 3, M-O). The colony size, colony number, and derived cell number of the $P 2 x 7-$ knockdown AML cells were also dramatically decreased, further indicating a remarkable inhibition of the self-renewal ability of LICs (Supplemental Figure 3, P and Q). These results imply that ATP/P2X7 signaling promotes the homing and self-renewal abilities of LICs, which further contribute to leukemogenesis.

$P 2 X 7$ is required for the proliferation of human $A M L$ cells. To further examine the role of P2X7 in human LICs, we first analyzed the mRNA levels of $P 2 X 7$ in human AML cells using TCGA database. Strikingly, the $P 2 X 7 \mathrm{mRNA}$ level in the AML cells was approximately 7-fold higher than that in the normal BM cells (Figure $4 \mathrm{~A}$ ), and the $P 2 X 7$ level was negatively correlated with the overall survival of AML patients (Figure $4 \mathrm{~B}$ ), indicating that P2X7 may be critical for leukemogenesis.

To further evaluate the functions of $\mathrm{P} 2 \mathrm{X} 7$ in human AML cells, we examined the $P 2 X 7$ levels in several human AML cell lines by quantitative RT-PCR and showed that $P 2 X 7$ was expressed at high levels in THP-1 (M5), U937 (M5), and MV4-11 (M5) cells but expressed at low levels in HL60 (M3), NB4 (M3), and K562 (CML) cells (Supplemental Figure 4A). We then constructed 2 shRNAs to knock down P2X7 in several human AML cell lines to evaluate its roles in human leukemia development. Both the shRNAs (sh-P2X7-1 and -2) efficiently downregulated the mRNA level of $P 2 X 7$ in THP- 1 cells, as measured by quantitative RT-PCR (Supplemental Figure 4, B and C). Knockdown of $P 2 X 7$ in 3 human AML cell lines, THP-1, U937, and MV4-11 cells, resulted in a marked decrease in cell proliferation in vitro at the indicated time points (Figure 4, C-E). We then transplanted the P2X7-knockdown THP-1 cells into NOD-SCID mice and found that leukemia development was notably delayed in these mice, as evidenced by the decreased leukemia cell frequency in peripheral blood (Supplemental Figure 4D), prolonged overall survival (32 or 33 vs. 23 days, Figure $4 \mathrm{~F}$ ), and the smaller size and lower weight of the spleens and livers (Supplemental Figure 4, E and F) in these mice compared with those of the control mice transplanted with the scrambled shRNA-treated THP-1 cells. 
A

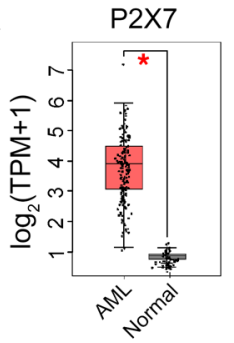

B

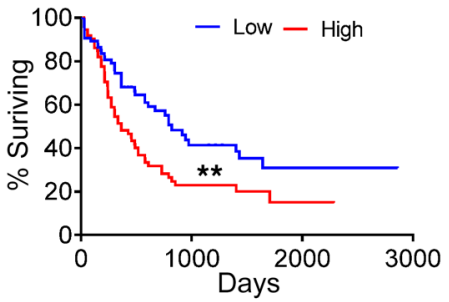

C

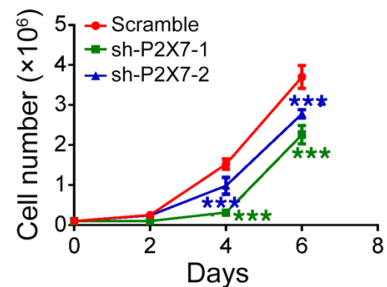

D

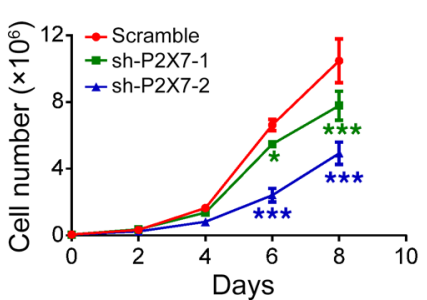

E

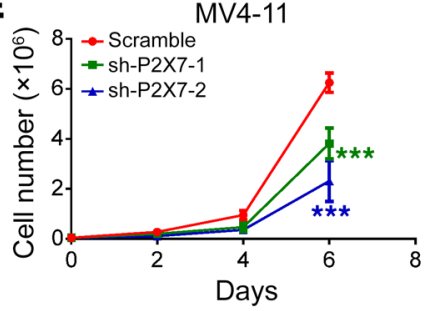

H

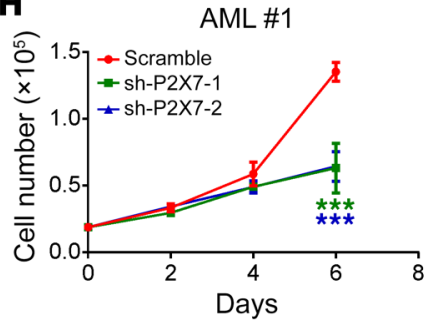

$\mathbf{F}$

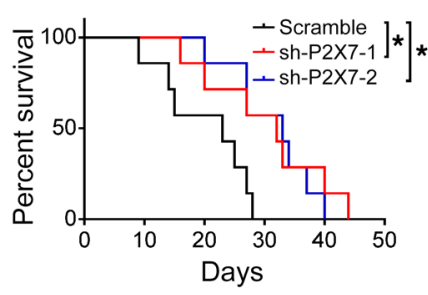

I

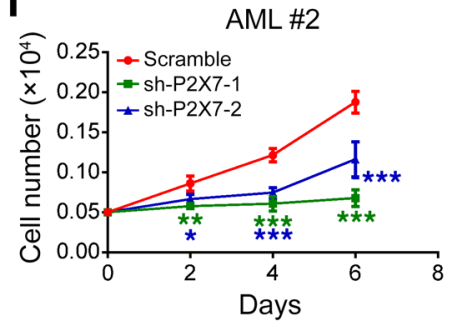

G

hLICs
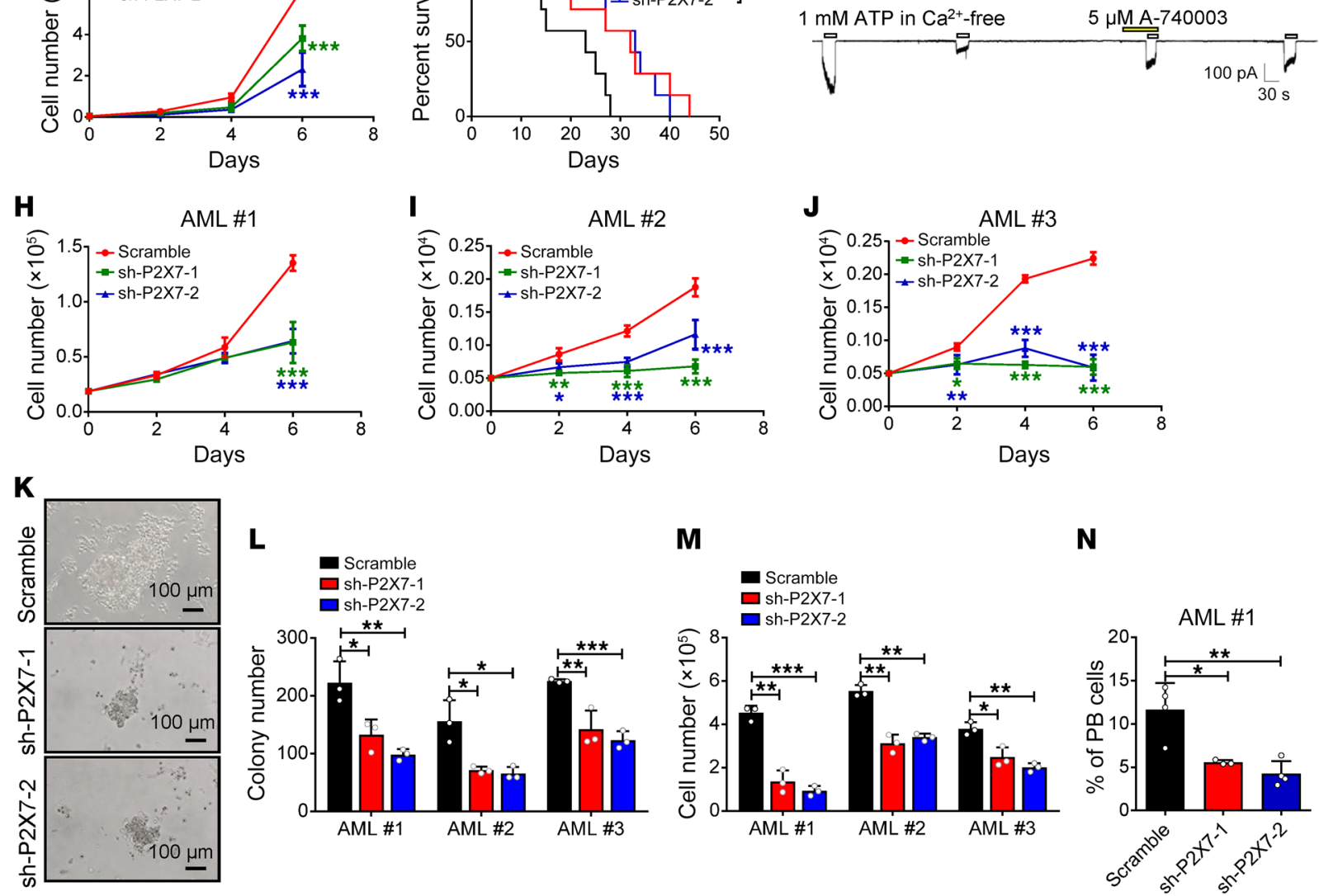

$\mathbf{L}$

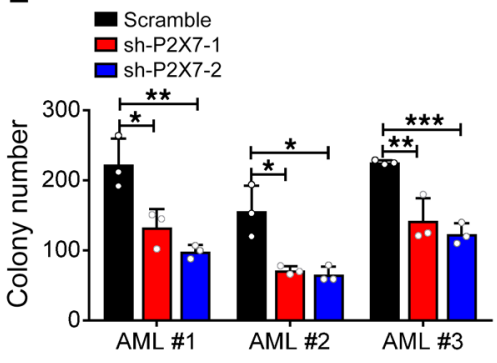

M

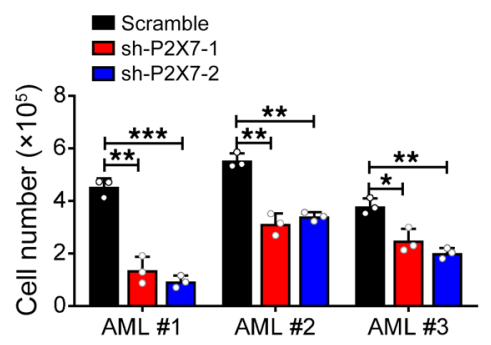

$\mathbf{N}$

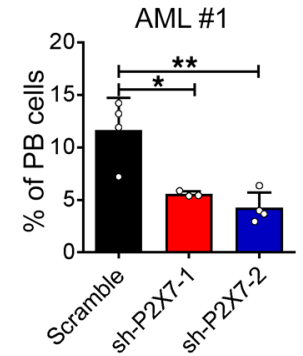

。

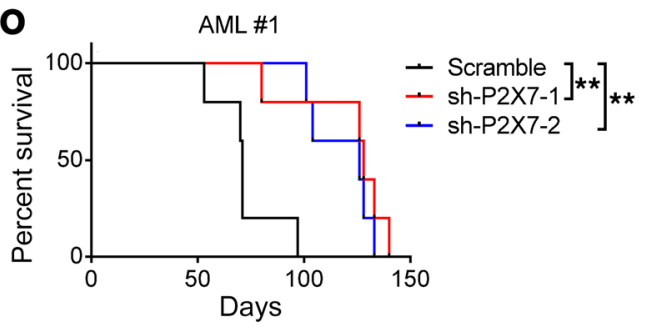

$\mathbf{P}$

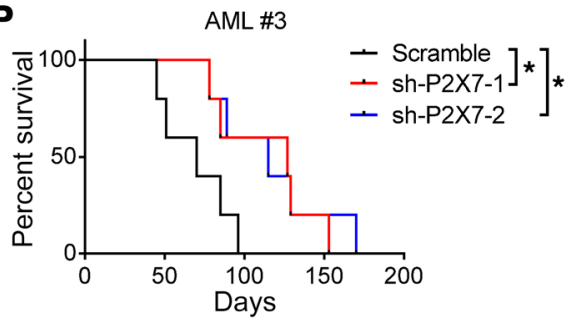

Figure 4. P2X7 is required for the proliferation of human AML cells. (A) P2X7 mRNA levels were analyzed in AML cells and normal BM cells from TCGA database (AML, $n=173$; normal $n=70$ ). ${ }^{*} P<0.05$ by Student's $t$ test. TPM, transcripts per million. (B) The relationship between $P 2 X 7$ level and survival of AML patients from TCGA database ( $n=75 /$ group). ${ }^{* *} P<0.01$ by log-rank test. (C-E) The numbers of THP-1, U937, and MV4-11 cells were calculated after infection with shRNAs targeting P2X7 (sh-P2X7-1 and -2) or scrambled shRNA $(n=3) .{ }^{*} P<0.05$, ${ }^{* *} P<0.001$ by 2-way ANOVA with Sidak's multiple-comparison test. (F) The overall survival was examined in the recipients transplanted with P2X7-knockdown (sh-P2X7-1 and -2 ) THP-1 cells and scrambled shRNA ( $n=7$ ). ${ }^{*} P<0.05$ by log-rank test. (G) P2X7-mediated influx of ions into human CD34+ LICs was measured upon the sequential treatment with ATP and the P2X7 antagonist A-740003 $(n=3)$. (H-J) The numbers of human primary AML cells were counted upon $P 2 X 7$ knockdown. Three patients' samples were examined (AML 1-3) $(n=3) .{ }^{*} P<0.05,{ }^{* *} P<0.01,{ }^{* * *} P<0.001$ by 2-way ANOVA with Sidak's multiple-comparison test. (K) Representative images of colonies derived from human AML cells upon the knockdown of $P 2 X 7$ by shRNAs (sh-P2X7-1 and -2) or those treated with scrambled shRNA control. (L and $M$ ) Colony numbers and derived total cell counts were examined in human AML cells upon the knockdown of $P 2 X 7$ by shRNAs (sh-P2X7-1 and -2 ) and scrambled shRNA ( $n=3)$. ${ }^{*} P$ $<0.05$, ${ }^{* *} P<0.01$, ${ }^{* *} P<0.001$ by 2 -way ANOVA with Sidak's multiple-comparison test. ( $\left.\mathbf{N}-\mathbf{P}\right)$ CD45+CFP+ human AML cells in the peripheral blood (N) (AML $1, n=5$ ) and the survival $(\mathbf{O}$ and $\mathbf{P})(A M L 1$ and $2, n=5$ ) of the recipients transplanted with $P 2 X 7-$ knockdown (sh-P2X7-1 and -2) human AML cells or scrambled shRNA are shown. PB, peripheral blood. ${ }^{*} P<0.05,{ }^{* *} P<0.01$ by 1-way ANOVA with Tukey's multiple-comparison test (N) or log-rank test $(\mathbf{O}$ and $\mathbf{P})$. 
We then further evaluated the role of $\mathrm{P} 2 \mathrm{X} 7$ in human primary AML cells and found that the $P 2 X 7$ mRNA level in LICs was approximately 7-fold higher than that in HSCs (Supplemental Figure 4G). Importantly, ATP supplementation efficiently induced the flux of ions into human CD34+ AML LICs, which was completely blocked by the P2X7 antagonist A-740003, as measured by whole-cell patch-clamp recording (Figure $4 \mathrm{G}$ ). Knockdown of $P 2 X 7$ in human CD34+ AML LICs resulted in an approximately $60 \%-80 \%$ reduction in cell growth in vitro (Figure $4, \mathrm{H}-\mathrm{J}$ ). An in vitro functional colony forming assay further showed that the P2X7-knockdown LICs generated approximately 40\%-60\% fewer colony numbers and 40\%-80\% fewer total cells than the LICs infected with the scrambled shRNA (Figure 4, K-M). Moreover, in vivo transplantation experiments revealed that $P 2 X 7$ knockdown led to a marked delay in AML development, as evidenced by the decreased percentages of leukemia cells in the peripheral blood (Figure $4 \mathrm{~N}$ ) and the significantly extended overall survival in the recipient mice transplanted with $P 2 X 7$-knockdown AML cells compared with those of the control mice (128 or 126 vs. 71 days in Figure 4O, and 127 or 115 vs. 70 days in Figure 4P). AML development in the recipient mice was confirmed based on the expression of several myeloid markers, such as CD33, CD15, and CD34, but not lymphoid markers, such as CD19 and CD20 (Supplemental Figure $4 \mathrm{H}$ ). These results indicate that $\mathrm{P} 2 \mathrm{X7}$ is critical for the leukemogenic capacities of human AML LICs.

$A T P / P 2 X 7$ signaling sustains LIC activities by activating the PHGDH pathway. To determine the underlying molecular mechanisms that control the homing and self-renewal activities of P2x7-null LICs, WT and P2x7-null LICs were analyzed by RNA sequencing. The RNA sequencing data showed that 672 or 576 genes were significantly up- or downregulated in the $P 2 x 7$-null group compared with the WT group (Supplemental Figure 5A). Among the top 15 significant differences in the Gene Ontology (GO) or Kyoto Encyclopedia of Genes and Genome (KEGG) analyses, $P 2 x 7$ has been shown to be involved in biological processes, such as cell migration, regulation of cell proliferation, cellular response to calcium ions, cell adhesion, and ATP metabolic process (Supplemental Figure 5B), or to be associated with several pathways, including oxidative phosphorylation, focal adhesion, and glycine, serine, threonine, and purine metabolism (Supplemental Figure $5 \mathrm{C}$ ). Because we noticed that several metabolic pathways (such as ATP metabolic process, oxidative phosphorylation, and glycine, serine, threonine, and purine metabolism) were markedly downregulated in the P2x7-null LICs and that $P 2 x 7$ deletion led to the loss of homing to the endosteal niche and self-renewal abilities of the LICs, we first examined the potential changes in several genes related to glycine, serine, and threonine metabolism (such as Phgdh, Psat1, and Shmt1), homing activities (such as Cxcr4, Cd44, Emb, Cc3cr1, and Vcam1), and self-renewal activities (such as Creb, Mef2c, Camk1d, Camk4, and Hoxb4) in the RNA sequencing data. This analysis showed that several key genes were indeed significantly decreased in the P2x7-null LICs (Supplemental Figure 5D). Consistently, quantitative RT-PCR further demonstrated that most of the key genes, including Phgdh, Psat1, Psph (related to glycine serine metabolism), Cxcr4, Selplg, Cd44 (related to homing), Creb, and Hoxb4 (related to self-renewal), were significantly downregulated in the $P 2 x 7$-null
LICs (Figure 5A). These results indicated that these genes might serve as downstream targets of P2X7.

Serine has been known to serve as a proteinogenic amino acid and the source of 1-carbon units important for de novo purine and deoxythymidine synthesis. Because PHGDH is the key rate-limiting enzyme involved in the first step of serine and glycine synthesis and has been reported to be critical for the development of several types of cancers $(51,52)$, we further examined the protein level of PHGDH. We found that the level of the PHGDH protein was markedly decreased in the P2x7-null LICs compared with the WT control cells (Figure 5B). This finding was consistent with the much lower level of serine in the P2x7-null LICs compared with that in the control LICs, as measured by liquid chromatographytandem mass spectrometry (LC-MS/MS) (Figure 5C). To further verify that $P h g d h$ is a downstream target gene of $\mathrm{P} 2 \mathrm{X} 7$, we overexpressed Phgdh in P2x7-null AML cells and transplanted these cells into lethally irradiated recipient mice. The overexpression of $P h g d h$ in the P2x7-null AML cells fully reversed the extended survival of the recipient mice to levels comparable to those of the recipient mice transplanted with WT leukemia cells (Figure 5, D and E, and Supplemental Figure 5, E and F). In addition, the overexpression of Phgdh had no effect on WT leukemia cell proliferation (Figure 5, D and E, and Supplemental Figure 5, E and F). Importantly, an in vitro Transwell assay also showed that overexpression of $P h g d h$ reversed the impaired migratory abilities of the $P 2 x 7$-deficient LICs (Supplemental Figure 5, G and H) or bulk AML cells (Supplemental Figure 5, I and J). In vivo homing analysis further revealed that the Phgdh-overexpressing P2x7-null AML cells exhibited enhanced homing activities compared with the P2x7-null AML cells; the homing activities of the Phgdh-overexpressing P2x7-null AML cells were comparable to those of the WT AML cells (Figure $5 \mathrm{~F}$ and Supplemental Figure 5K). Consistently, the CXCR4 protein level was much lower in both P2x7-null LICs and bulk BM cells than that of WT controls, which could be significantly upregulated upon the overexpression of Phgdh in P2x7-null leukemia cells (Figure 5G and Supplemental Figure 5, L-N), indicating that PHGDH regulates the homing activities of LICs. To further confirm that serine metabolism plays a role in leukemogenesis, an in vitro functional colony forming assay was performed in the presence or absence of serine and glycine. Interestingly, the colony size, colony numbers, and derived cell numbers of the leukemia cells were significantly decreased upon the depletion of serine and glycine (Figure 5, H and I). In vivo transplantation experiments further showed that the depletion of serine/glycine resulted in a notable delay in leukemia development, as exhibited by the decreased frequency of AML cells in the peripheral blood and the prolonged overall survival of the recipient mice (Figure 5, J and K).

CREB signaling maintains PHGDH levels to enhance leukemogenesis. Because many studies have shown that $\mathrm{P} 2 \mathrm{X} 7$ mediates the influx of calcium and the efflux of potassium (41), and because our RNA sequencing data also revealed a significant reduction in the cellular response to calcium ions (Supplemental Figure 5A), we further measured the calcium influx capacity of the P2x7-null LICs using the calcium indicator Fura-2 AM. Interestingly, the constitutive calcium influx was markedly decreased in both the $P 2 x 7$-null LICs (Figure 6A) and bulk AML cells (Supplemental Figure 6A) compared with that of the WT cells. Because the transcription 
A

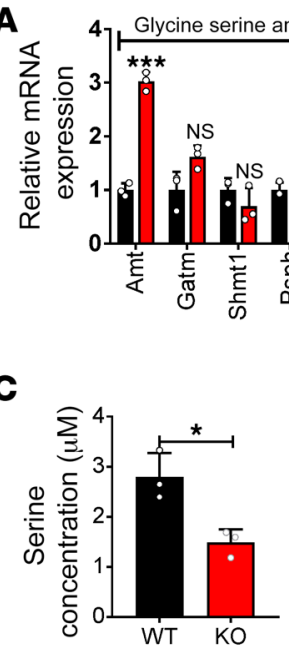

G

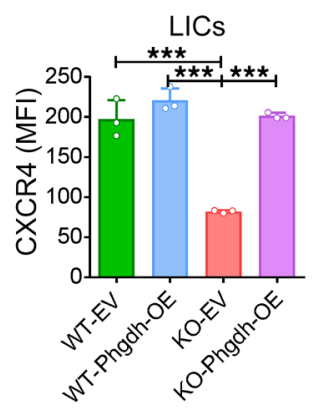

D

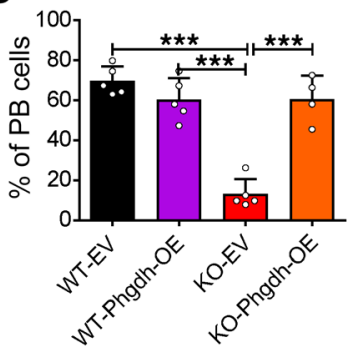

Self-renewal

\section{$\mathbf{B}_{\mathrm{KO}}^{\mathrm{W}}$

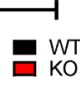

NS
B

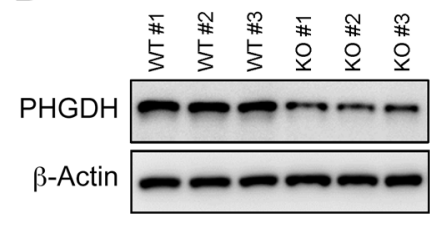

H

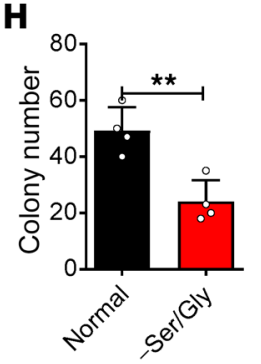

E

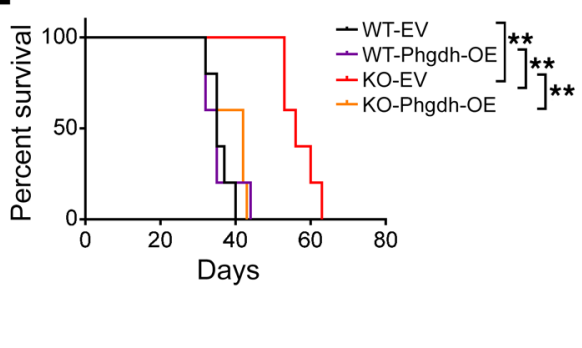

F

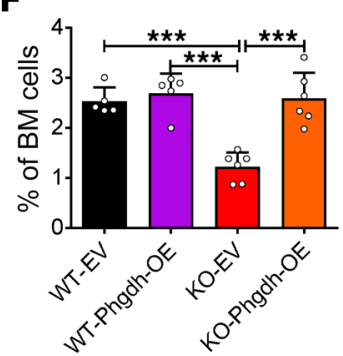

I

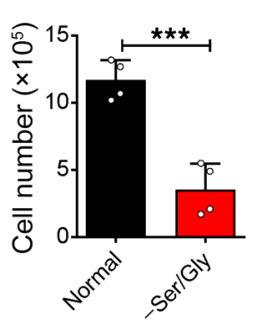

J

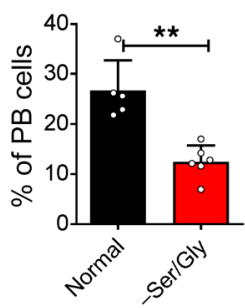

$\mathbf{K}$

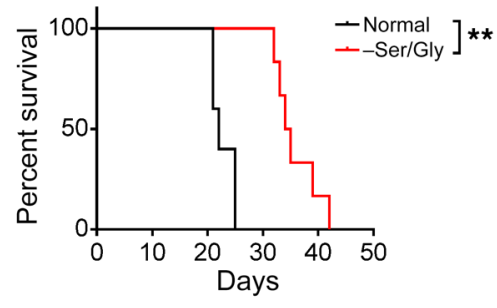

Figure 5. ATP/P2X7 signaling maintains LIC activities by activating the PHGDH pathway. (A) Potential candidates related to glycine, serine, and threonine metabolism, homing, and self-renewal were examined in WT and P2x7-KO Mac-1+c-Kit ${ }^{+}$LICs by quantitative RT-PCR $(n=3)$. ${ }^{*} P<0.05,{ }^{* *} P<0.01$, ${ }^{* * *} P<0.001$ by 2 -way ANOVA with Sidak's multiple-comparison test. (B) Protein levels of PHCDH were determined in WT and P2x7-KO Mac-1+C-Kit ${ }^{+}$LICs. (C) Serine levels were measured in WT and P2x7-KO BM leukemia cells by LC-MS/MS $(n=5)$. ${ }^{*} P<0.05$ by Student's $t$ test. (D and E) The leukemia cell (YFP'mCherry $)$ frequencies in the peripheral blood (D) $\left(n=5 ;{ }^{* * *} P<0.001\right.$ by 1 -way ANOVA with Tukey's multiple-comparison test) and overall survival (E) ( $n=5$; ${ }^{* *} P<0.01$ by log-rank test) were compared among the recipients transplanted with WT, $P 2 \times 7-\mathrm{KO}$, Phgdh-overexpressing WT, or $P 2 \times 7-K O$ leukemia cells. (F) The frequency of homed WT, P2x7-KO, Phgdh-overexpressing WT, or P2x7-KO leukemia cells (CFSE') in the BM of the recipients 16 hours after transplantation $(n=5) .{ }^{* *} P<0.001$ by 1-way ANOVA with Tukey's multiple-comparison test. (C) The mean fluorescence intensities (MFIs) of CXCR4 in WT, P2x7-KO, Phgdh-overexpressing WT, or P2x7-KO Mac-1+c-Kit ${ }^{+}$LICs $(n=3) .{ }^{* *} P<0.001$ by 1-way ANOVA with Tukey's multiple-comparison test. (H and I) The colony formation abilities of leukemia cells were determined in the presence or absence of serine and glycine (normal or -Ser/Gly). Colony numbers $(\mathbf{H})$ and derived total cell counts (I) were calculated $(n=3) .{ }^{* *} P<0.01,{ }^{* * *} P<0.001$ by Student's $t$ test. (J) Leukemia cells were cultured with or without serine and glycine (normal or -Ser/Gly) for 48 hours, followed by transplantation into the recipient mice. Shown are the leukemia cell frequencies in the peripheral blood of recipients 3 weeks after transplantation $(n=5-6)$. ${ }^{*} P<0.01$ by Student's $t$ test. (K) Overall survival was determined in the leukemic mice in panel $\mathbf{J}(n=5-6) .{ }^{* *} P<0.01$ by log-rank test. EV, empty vector; OE, overexpressing; PB, peripheral blood.

factor CREB serves as a key mediator of calcium signaling and its mRNA level was also markedly decreased upon $P 2 x 7$ deletion, it is possible that CREB may act as an upstream target of $\mathrm{P} 2 \mathrm{X} 7$. Consistently, the protein levels of phospho-CREB (p-CREB) (S133) and CREB in the P2x7-null LICs were significantly reduced compared with those in the WT control LICs (Figure 6B). Compared with the $P 2 x 7$-null control cells, $P 2 x 7$-null AML cells overexpressing Creb accelerated the development of leukemia and reversed the phenotypes resulting from $P 2 x 7$ deletion, as evidenced by the increased frequency of leukemia cells in the peripheral blood (Figure 6, C and D), the enhanced infiltration in spleens and livers (Supplemental Figure 6, B and C), and the reduced overall survival; these parameters were comparable to those observed in the mice transplanted with WT AML cells (Figure 6E). The levels of Creb in the
WT, $P 2 x 7$-null, Creb-overexpressing WT, or Creb-overexpressing P2x7-null AML cells were confirmed by quantitative RT-PCR (Supplemental Figure 6D). The overexpression of Creb in the WT AML cells had no effect on leukemogenesis (Figure 6, C and D, and Supplemental Figure 6, B and C). Moreover, the overexpression of Creb also upregulated the protein level of CXCR4 in both the P2x7-null LICs and bulk AML cells, and these levels were comparable to that observed in the WT cells (Figure 6, F and G, and Supplemental Figure 6, E and F). These results suggest that CREB-mediated calcium signaling is required for the leukemogenic activities of $\mathrm{P} 2 \mathrm{X} 7$.

To determine whether P2X7 is regulated by CREB-mediated signaling, the mRNA level of Phgdh was evaluated in both Creb-overexpressing WT and P2x7-null AML cells by quantita- 
A

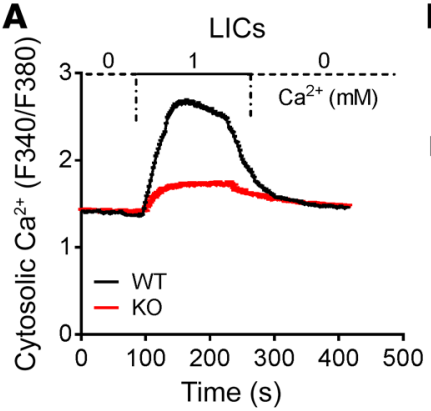

B

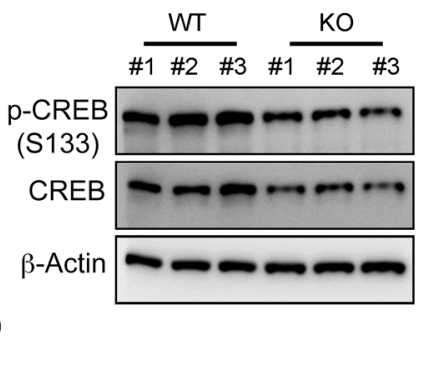

C

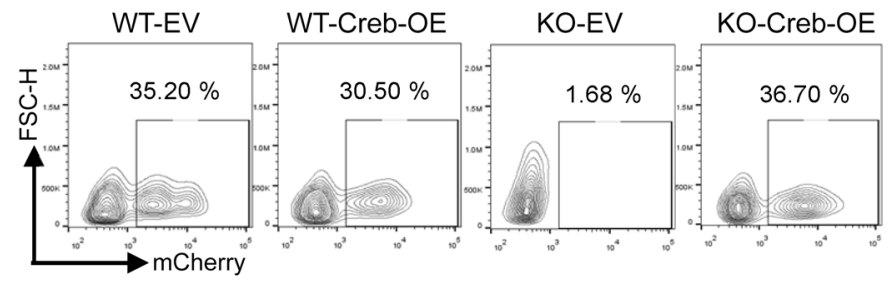

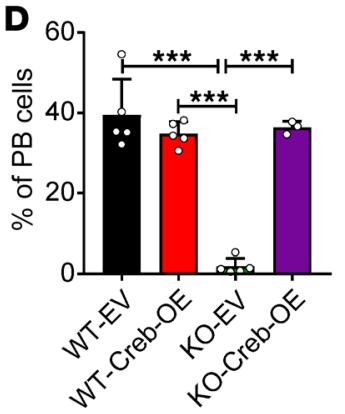

E

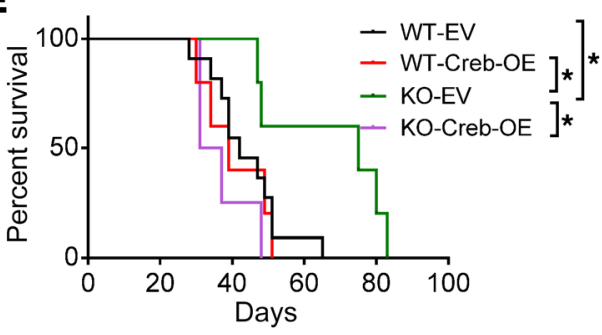

$\mathbf{F}$
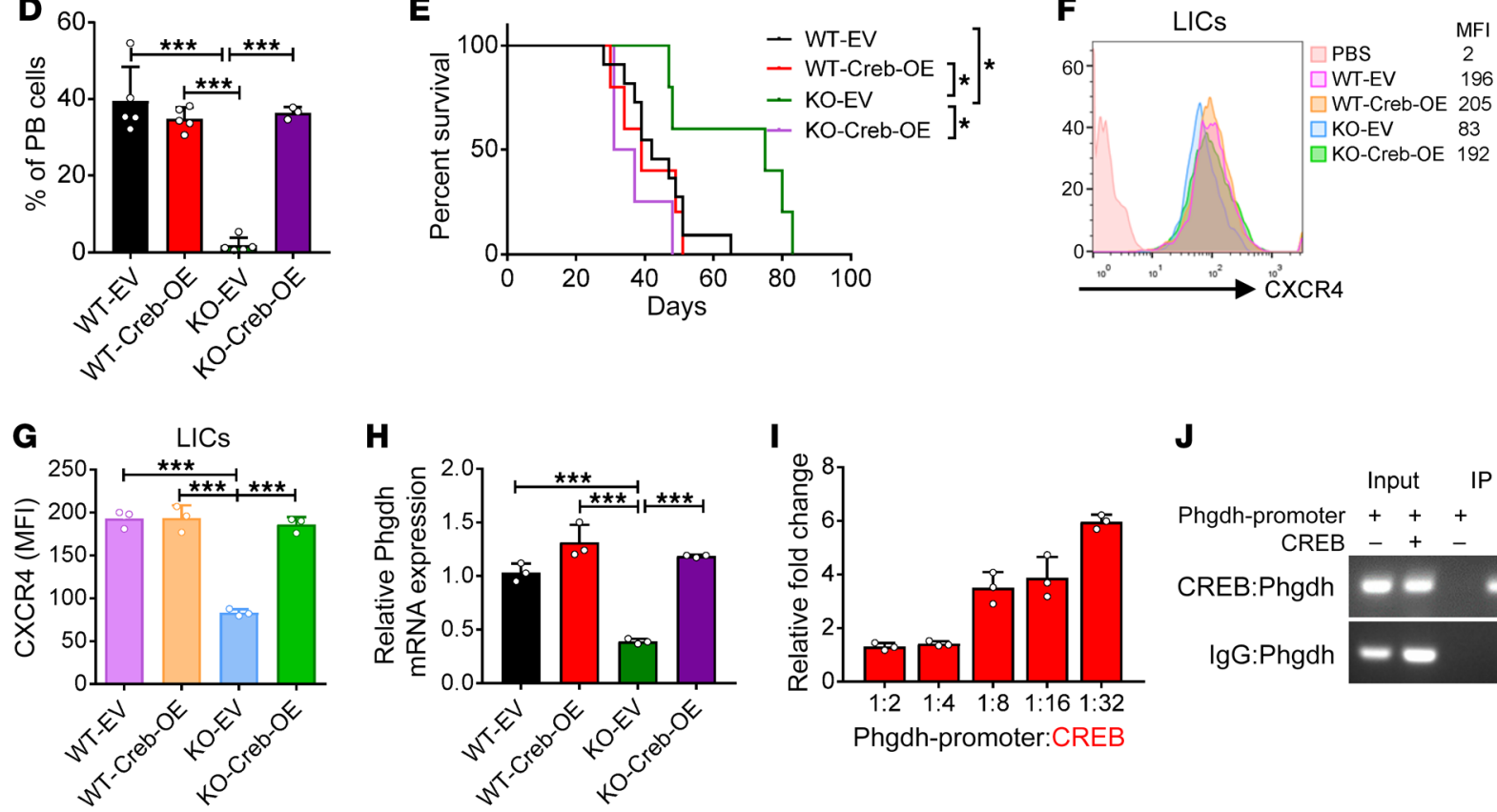

J

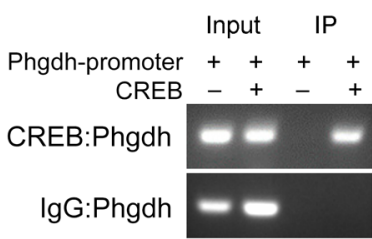

Figure 6. CREB signaling maintains PHGDH levels to enhance leukemogenesis. (A) The constitutive calcium influx of WT and $P 2 x 7-K O$ Mac- $1^{+} c-K i t^{+} L I C s$ was measured using Fura-2 AM ( $n=205$ cells; $0=\mathrm{Ca}^{2+}$-free solution; 1 = solution with 1 mM Ca ${ }^{2+}$ ). (B) The protein levels of phospho-CREB (S133) and CREB were determined in both WT and P2x7-KO Mac-1+c-Kit ${ }^{+}$LICs by Western blot. (C) Representative flow cytometric analysis of leukemia cells (YFP+mCherry in the peripheral blood of recipient mice transplanted with WT, P2x7-KO, Creb-overexpressing WT, or P2x7-KO AML cells. (D) Quantification of data in panel C $(n=5){ }^{* * *} P<0.001$ by 1-way ANOVA with Tukey's multiple-comparison test. (E) The overall survival was compared among the mice transplanted with WT, $P 2 x 7-\mathrm{KO}$, Creb-overexpressing WT, or $P 2 x 7-\mathrm{KO}$ AML cells $(n=5)$. ${ }^{*} P<0.05$ by log-rank test. (F) The expression levels of CXCR4 in WT, $P 2 x 7-K 0$, Creb-overexpressing WT, or P2x7-KO Mac- $1^{+} \mathrm{C}-\mathrm{Kit}{ }^{+}$LICs were determined by flow cytometry. (G) Quantification of the mean fluorescence intensities (MFIs) in panel $\mathbf{F}(n=3)$. ${ }^{* *} P<0.001$ by 1-way ANOVA with Tukey's multiple-comparison test. (H) The mRNA levels of Creb in WT, $P 2 x 7$-KO, Creb-overexpressing WT, or P2x7-KO AML cells were measured by quantitative RT-PCR $(n=3)$. ${ }^{* *} P<0.001$ by 1 -way ANOVA with Tukey's multiple-comparison test. (I) A luciferase reporter assay was used to evaluate the transcriptional activity of CREB on the promoter of Phgdh ( $n=3-4)$. (J) The binding of CREB to the Phgdh promoter region was determined by chromatin immunoprecipitation (ChIP) assay. EV, empty vector; OE, overexpressing; PB, peripheral blood.

tive RT-PCR; the results showed that Creb overexpression indeed resulted in a significant increase in the Phgdh levels (Figure 6H). By using a dual luciferase reporter assay, we further demonstrated that Creb could directly bind to the Phgdh promoter region and transactivate the Phgdh expression in a dose-dependent manner (Figure 6I); these findings were further validated by subsequent ChIP analysis with an antibody against CREB (Figure 6J). Consistently, compared with the knockdown control, knockdown of $P 2 x 7$ in primary mouse AML cells also led to a notable reduction in the protein levels of p-CREB, CREB, and PHGDH (Supplemental Figure $6 G$ ). These results demonstrated that ATP/P2X7 signaling enhances the homing and self-renewal capacities of LICs through CREB/PHGDH signaling pathways.
To further verify that P2X7 exerts its leukemogenic effect on human LICs via pathways similar to those used by murine LICs, we examined the protein levels of p-CREB, CREB, and PHGDH in both the AML cell lines and primary samples. This analysis showed that $P 2 X 7$ knockdown led to a marked reduction in CREB/ PHGDH signaling (Supplemental Figure 7, A and B). Similarly to the murine $P 2 x 7$-null AML cells, the $P 2 X 7$-knockdown human primary AML cells also had lower levels of serine than the primary AML cells infected with the scrambled shRNA, further indicating that impaired serine metabolism may inhibit AML cell proliferation (Supplemental Figure 7C). Depletion of serine and glycine from the culture medium also resulted in an approximately $30 \%-$ $70 \%$ reduction in the growth of several AML cell lines, THP-1, 

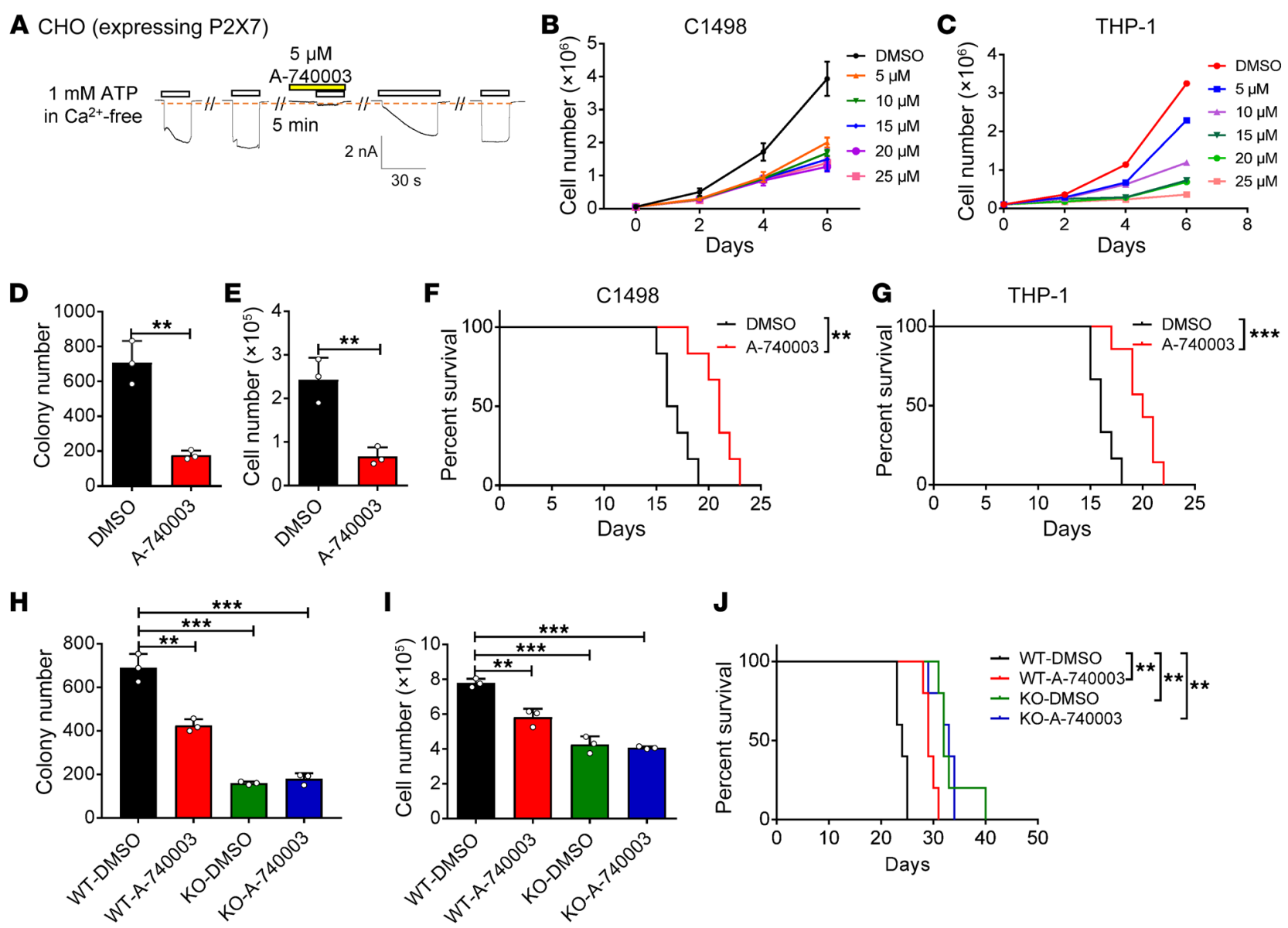

Figure 7. Targeting leukemia development by suppressing ATP/P2X7 signaling. (A) The P2X7-mediated influx of ions in $\mathrm{CHO}$ cells overexpressing rat $P 2 \times 7$ was measured upon sequential treatments with extracellular ATP and the P2X7 antagonist A-740003 by whole-cell patch-clamp recoding ( $n=3$ ). (B and C) C1498 cells (B) and THP-1 cells (C) were treated with the indicated doses of A-740003 and the numbers were calculated $(n=3)$. ( $\mathbf{D}$ and $\mathbf{E})$ Colony numbers (D) and derived total cell counts (E) were determined in THP-1 cells treated with A-740003 $(10 \mu \mathrm{M})$ and DMSO $(n=3)$. ${ }^{* *} P<0.01$ by Student's $t$ test. $(\mathbf{F}$ and G) The overall survival was compared in the recipient mice transplanted with C1498 cells (F) or THP-1 cells (G), followed by the treatments with A-740003 and DMSO $(n=5) .{ }^{* *} P<0.01,{ }^{* *} P<0.001$ by log-rank test. ( $\mathbf{H}$ and $\left.\mathbf{I}\right)$ Colony numbers $(\mathbf{H})$ and derived total cell counts $(\mathbf{I})$ of WT and $P 2 \times 7-K 0$ murine AML cells were measured upon the treatments with A-740003 $(10 \mu \mathrm{M})$ and DMSO $(n=3)$. ${ }^{*} P<0.01,{ }^{* * *} P<0.001$ by 1-way ANOVA with Tukey's multiple-comparison test. (J) The overall survival was compared in the recipient mice transplanted with WT and $P 2 \times 7-K O$ AML cells, followed by the treatments with A-740003 and DMSO 7 days after transplantation ( $50 \mathrm{mg} / \mathrm{kg}$ every other day for 2 weeks) $(n=5){ }^{* *} P<0.01$ by log-rank test.

U937, and MV4-11 cells (Supplemental Figure 7, D-F). Moreover, an in vitro functional colony assay revealed that the numbers of colonies and the total cells derived from the human primary AML cells were reduced to approximately $50 \%-70 \%$ of those derived from the control cells when serine and glycine were depleted from the methylcellulose medium (Supplemental Figure 7, G and H). We also cultured human cord blood $\mathrm{CD}_{3} 4^{+}$cells with and without serine/glycine in vitro and found that impaired serine/glycine metabolism did not affect the proliferation of normal $\mathrm{CD} 34^{+}$cells (Supplemental Figure 7, I and J), indicating such an effect may be specific to AML cells.

Consistently, we also revealed that the ATP level in the BM fluid of human AML patients was much higher compared with healthy donors (Supplemental Figure 7K), which was consistent with the finding in the murine model. Interestingly, it seems that the ATP level in several subtypes (M2, M3, and M5) of AML were also much higher than the normal ones (Supplemental Figure 7L). We further analyzed the expression of CREB, CXCR4, and
PHGDH in normal and AML data sets, which showed that CXCR4 and CREB, but not PHGDH, were upregulated in AML patients and consistent with the expression of P2X7 in human AML (Supplemental Figure 7, M-O). Although the mRNA level of $P H G D H$ in AML patients was not consistent with that of $P 2 X 7$ and was relatively lower than that of normal ones, the overall survival was inversely correlated with $P H G D H$ expression and similar to that of P2X7 (Supplemental Figure 7P), indicating that PHGDH may still serve as a critical oncogene in leukemia development. Meanwhile, we also found that the protein level of PHGDH was notably increased in human AML samples compared with that of the normal BM cells, indicating that posttranslational modification may be important for the high protein level and function of PHGDH in leukemogenesis (Supplemental Figure 7, Q and R).

Targeting AML development by suppressing ATP/P2X7 signaling. To determine whether the blockade of ATP/P2X7 signaling can inhibit AML development, we extensively evaluated the potential therapeutic effect of a specific antagonist of P2X7, A-740003, 
both in vitro and in vivo. Similar to the experiments mentioned above (Figure 2E and Figure $4 \mathrm{G}$ ), we tested the inhibitory effect of A-740003 by analyzing $P 2 x 7$-overexpressing $\mathrm{CHO}$ cells by wholecell patch-clamp technology. This analysis showed that the ion flux induced by extracellular ATP could be completely inhibited by $5 \mu \mathrm{M}$ A-740003 and gradually recovered thereafter (Figure 7A). A-740003 sufficiently inhibited the in vitro proliferation of both the murine (C1498, Figure 7B) and human (THP-1, Figure 7C) AML cell lines in a dose-dependent manner. An in vitro functional colony forming assay further showed that A-740003 treatment notably impaired the colony forming capacity of THP-1 cells, as exhibited by the smaller colony size and fewer numbers of total cells after A-740003 treatment than after control treatment (Supplemental Figure 8A and Figure 7, D and E). These results prompted us to evaluate the potential therapeutic effects of A-740003 in vivo. Recipient mice were transplanted with either C1498 or THP-1 cells and intraperitoneally injected with A-740003 for 2 weeks (Supplemental Figure 8B). Interestingly, A-740003 treatment resulted in a significant reduction in the frequency of leukemia cells in the peripheral blood (Supplemental Figure 8, C-F) and a notably extended survival time of the recipient mice compared with the control mice (Figure 7, F and G), indicating that A-740003 can indeed be used to effectively target AML cells.

We then examined the therapeutic effect of A-740003 in an MLL-AF9-induced aggressive AML model. An in vitro functional colony formation analysis revealed that A-740003 treatment caused an approximately $30 \%$ reduction in colony formation and cell count compared with the control treatment (Figure 7, $\mathrm{H}$ and I). More importantly, after 2 weeks of intraperitoneal injection with A-740003, the recipient mice transplanted with the WT AML cells, but not the P2x7-null AML cells, exhibited a notable delay in leukemogenesis compared with the control-treated mice, as evidenced by the decreased percentages of both $\mathrm{YFP}^{+}$leukemia cells in the peripheral blood (Supplemental Figure 8, G and $\mathrm{H}$ ) and the significantly extended overall survival (Figure 7J). There was no difference in early/late apoptosis in both murine and human primary AML cells (Supplemental Figure 8, I-L), which was consistent with the findings in WT and P2x7-KO AML cells (Supplemental Figure 3, J and $\mathrm{K}$ ). These results indicate the antagonist A-740003 may target AML development by inhibiting the selfrenewal ability LICs. A-740003 treatment also significantly reduced the $\mathrm{p}-\mathrm{CREB}, \mathrm{CREB}$, and $\mathrm{PHGDH}$ protein levels in the WT AML cells but not in the P2x7-null AML cells, which may contribute to the marked delay in leukemogenesis (Supplemental Figure $8 \mathrm{M})$. Consistently, an in vitro colony formation assay showed that the colony sizes, numbers, and derived total cell counts of the human primary AML cells were markedly reduced upon the addition of A-740003 to the methylcellulose medium (Supplemental Figure 8, N-P). CREB phosphorylation and PHGDH levels were significantly reduced in human AML samples upon A-740003 treatment (Supplemental Figure 8Q).

Alternatively, we treated both murine and human primary AML cells with different doses of ATP in vitro and revealed that low doses of ATP (10-100 $\mu \mathrm{M})$ indeed could enhance the proliferation of leukemia cells of WT, but not $P 2 x 7-\mathrm{KO}$, murine leukemia cells (Supplemental Figure 9, A and B) or human ones (Supplemental Figure 9, C-E). In contrast, a high dose of ATP (1 mM) inhibited leukemia cell growth (Supplemental Figure 9, A-E), which might have resulted from nonspecific cytotoxicity (53-55). However, apoptosis (Supplemental Figure 9, F-J) and the cell cycle (Supplemental Figure 9, K-O) were not altered upon treatment with low-dose ATP (the changes in the high-dose group may be due to nonspecific cytotoxicity), indicating that ATP-mediated signaling may mainly sustain the self-renewal ability of leukemia cells and blockade of the ATP/P2X7 pathway is a potential strategy for leukemia treatment.

\section{Discussion}

In current study, we show a unique pattern of ATP distribution in the leukemic BM, as indicated by a genetically encoded ATP sensor (iATPSnFR), namely, the endosteal niche has a much higher ATP level than the vascular niche. The high levels of ATP can mediate the flux of calcium via the ion channel P2X7 to sustain the homing and self-renewal capacities of LICs. Calcium signaling further enhances the phosphorylation of CREB, which transactivates PHGDH to maintain the leukemogenic activities of LICs. Blocking ATP/P2X7 signaling with the specific antagonist A-740003 efficiently delays leukemia development both in vitro and in vivo (Supplemental Figure 9P). Our studies show that ATP may serve as an important niche factor that participates in the control of the fates of LICs via activation of P2X7, which may be an ideal therapeutic target in LICs or other types of cancer stem cells.

Increasing evidence shows that BM niches play key roles in sustaining LIC activities. However, the mechanisms by which niche factors affect the fates of different types of LICs remain elusive. Here, we provide unexpected data showing that ATP levels are markedly increased in leukemic BM niches compared with normal $\mathrm{BM}$ niches and support the homing and self-renewal abilities of LICs, which may be derived from the remodeling of the leukemic niche due to enhanced hypoxia or stress during leukemogenesis. Strikingly, we show that ATP levels in the leukemic BM niche are in the range of $59-723 \mu \mathrm{M}$, which is approximately 950-fold higher than that of the control (Figure 1A). Therefore, it is possible that such high ATP levels in leukemic BM niches may further induce P2X7-mediated ion flux to enhance AML development, although $\mathrm{P} 2 \mathrm{X} 7$ has been shown to have a notably high threshold for activation by ATP (ranging from 0.1 to $1.0 \mathrm{mM} \mathrm{ATP)} \mathrm{(56-58).} \mathrm{Moreover,}$ based on the ATP sensor (iATPSnFR), we establish a sensitive method for monitoring the dynamic changes in ATP levels both in vitro and in vivo; this ATP sensor will be useful to precisely determine the ATP levels in the different leukemic BM niches.

In the current study, we also showed that the endosteal niche has a much higher ATP level than the vascular niche, indicating that the osteoblasts in the niche may generate more extracellular ATP during leukemia development; however, other niche cells, such as osteoclasts, may also be involved in the secretion of ATP. It is also possible that LICs autonomously and substantially contribute to the extracellular ATP pools to maintain their leukemic capacities. Currently, extracellular ATP is reported to be mainly secreted by infected cells or cells undergoing certain stresses, and this secretion is regulated via pannexin 1 (Panx1) channels or connexin $43(\mathrm{Cx} 43)$ hemichannels $(46,47)$. Whether Panx1- or Cx43-mediated ATP secretion contributes to the enhanced ATP level in the leukemic BM niches requires further investigation. 
The identification of the main ATP-producing cell types and the underlying mechanisms in the $\mathrm{BM}$ niches during leukemogenesis will benefit the development of potential strategies for the eradication of LICs by blocking the interaction between LICs and niche cells or their regulatory networks. In the current study, we have shown that a genetically encoded ATP sensor (iATPSnFR) was able to differentially characterize the unique ATP distribution in the different leukemic BM niches. This potent tool may be useful for identifying the particular ATP-producing cells in leukemic BM niches or in other cancer microenvironments.

Previous studies have shown that extracellular ATP seems to be able to inhibit the proliferation of cancer cells, including prostate cancer, bladder cancer, and certain types of AML (such as the M3 type) (27, 29, 59). In addition, some studies have shown that ATP-mediated purinergic signaling can promote the growth of cancer cells, such as B-CLL, breast cancer, and cervical cancer $(16,60-$ 62). These seemingly contradictory results may be due to the capacity of ATP to bind to many different P2X or P2Y members. Because individual P2R members have very different functions in different physiological or pathological processes, the effects of ATP may primarily depend on the expression levels of $\mathrm{P} 2 \mathrm{R}$ members and their combined functions in certain cell types. Consistently, Casati et al. showed that HSCs express high levels of P2X1, -4, and -7 and that ATP sustains the size of the HSC population under stressful conditions mainly via $\mathrm{P} 2 \mathrm{X} 1$ and $\mathrm{P} 2 \mathrm{X} 4$ but not via $\mathrm{P} 2 \mathrm{X} 7$. P2X1 and $\mathrm{P} 2 \mathrm{X} 4$ have a synergistic effect in maintaining the HSC pool (25). P2y14-KO mice have markedly decreased HSC numbers after radiation stress, which may be due to the increased ROS and p16 levels (63). Therefore, it will be very important to examine the detailed roles of the individual P2Rs by using genetic tools, such as P2R-KO mice, together with treatments with nucleotides or specific P2R inhibitors.

In the present study, we generated $P 2 x 7-\mathrm{KO}$ mice and demonstrated that $\mathrm{P} 2 \mathrm{X} 7$ serves as an oncogene that enhances the homing and self-renewal abilities of LICs, which indicates that ATP-mediated purinergic signaling has different effects via individual P2X members. Interestingly, Salvestrini et al. reported that UTPP2Y2R/P2Y4R-mediated purinergic signaling sufficiently inhibits AML cell growth and homing to the BM. In contrast, these authors also provided preliminary and intriguing data indicating that high ATP levels may downregulate P2X7 expression to suppress AML cell proliferation in a negative feedback loop (64); however, whether ATP suppresses the expression of other P2X members to inhibit leukemia development remains unclear. Ectopic overexpression of $\mathrm{P} 2 \mathrm{X} 7$ is also capable of accelerating leukemia development (37). These results are consistent with our findings showing that P2X7 enhances leukemogenesis in a P2x7-KO AML model and suggest that it is critical to elucidate the precise roles of P2Rs by using combinational approaches with genetic animal models and treatments with nucleotides or specific inhibitors of P2Rs.

It has been shown that the intensity of the ATP stimulus may play a key role in regulating the ion channel or large pore functions of P2X7 to regulate its dual functions of promoting cellular survival and inducing cytotoxicity. How these 2 opposing effects are tightly regulated is still not clear, although studies have indicated that these effects may be controlled by the release of MMP2 or other unknown mechanisms (65). Additionally, the mechanism by which P2X7 regulates leukemia development remains elusive. Increas- ing evidence has indicated that $\mathrm{P} 2 \mathrm{X} 7$ may promote cell cycle progression to enhance leukemogenesis, as shown in a $P 2 x 7$-overexpressing model (37). It is also possible that calcium influx may be involved in P2X7-dependent leukemogenesis, since calcium signaling may be important for leukemia development (66). It has been reported that the hyposensitive N187D P2X7 mutant leads to a notable proliferative advantage of $\mathrm{K} 562$ cells both in vitro and in vivo, further indicating that the P2X7-mediated ion influx may be essential for leukemogenesis (67). Consistently, in the present study, we showed that the constitutive calcium influx is dramatically decreased in the P2x7-null LICs, which further results in a marked reduction in the phosphorylation of CREB. More interestingly, we further revealed that CREB can directly transactivate PHGDH expression, which is the key rate-limiting enzyme for serine/glycine synthesis. P2x7-null AML cells exhibit reduced capacities to mobilize and home to the BM niche, which is consistent with previous reports showing that $\mathrm{P} 2 \mathrm{X} 7$ is required for HSC mobilization to the peripheral blood upon G-CSF stimulation (68). Therefore, we provide what we believe is the first body of evidence showing a direct link between P2X7-mediated calcium signaling and serine/glycine metabolism for the maintenance of the homing and self-renewal abilities of LICs.

In conclusion, we have provided several lines of unexpected evidence showing that ATP levels are markedly increased and much higher in the endosteal niche than in the vascular niche during leukemogenesis. The high activation of the ATP/P2X7mediated CREB/PHGDH signaling pathways maintains the homing and self-renewal capacities of LICs. These findings provide a potential niche factor-related target to cure leukemia or other cancers. Further efforts are planned to evaluate therapeutic strategies that combine antagonist administration and serine restriction for the treatment of AML in vivo.

\section{Methods}

RNA sequencing. WT and P2x7-KO Mac- $1^{+} \mathrm{c}-\mathrm{Kit}^{+} \mathrm{LICs}$ were sorted by flow cytometry, followed by the extraction of total RNA and analysis by RNA sequencing at NovelBio Co., Ltd. A GO enrichment analysis was performed by Bioconductor package top GO (https://www. bioconductor.org/). The enrichment analysis of the KEGG pathway was conducted by Bioconductor package GSEA Base. We have deposited the RNA sequencing data in the NCBI Gene Expression Omnibus repository (GEO GSE162468).

Study approval. Human BM mononuclear cells of AML samples were provided by the Department of Hematology at Xinhua Hospital and the Sixth People's Hospital, Shanghai Jiao Tong University School of Medicine. Patients' written informed consent was obtained and approved by the Ethics Committee for Medical Research (IRB) of Shanghai Jiao Tong University School of Medicine.

All the other experimental details can be found in the supplemental material.

\section{Author contributions}

$\mathrm{XH}, \mathrm{JW}, \mathrm{HS}, \mathrm{YY}$, and JZ designed the experiments, performed experiments, analyzed data, and wrote the manuscript. $\mathrm{XY}, \mathrm{DH}$, XZ, XL, Y Zou, CC, ZY, LX, Y Zhang, LL, SL, and Y Zhao performed experiments, provided reagents, and helped with the manuscript preparation. 


\section{Acknowledgments}

This work was supported by grants from the National Basic Research Program of China (2019YFA0801800, 2018YFA0107000, and 2019YFA0904800), the National Natural Science Foundation of China (NSFC) (81825001, 81570093, 31971052, 81900147, 31722033, and 31671484), the innovative group of the NSFC (grant 81721004), the Shanghai Science and Technology Commission (19XD1422100, 20ZR1430900, 20JC1410100, and 17ZR1415500), and the Research Unit of New
Techniques for Live-cell Metabolic Imaging (Chinese Academy of Medical Sciences; 2019RU01, 2019-I2M-5-013).

Address correspondence to: Junke Zheng, 280 South Chongqing Road, Shanghai 200025, China. Phone: 86.21.63846590; Email: zhengjunke@shsmu.edu.cn. Or to: Ye Yu, 639 Long-Mian Road, Nanjing 211198, China. Phone: 86.25.86185472; Email: yuye@cpu. edu.cn. Or to: Hongfang Shao, 600 Yishan Road, Shanghai 200233, China. Phone: 86.25.64369181; Email: hongfangshao@sina.com.
1. Bonnet D, Dick JE. Human acute myeloid leukemia is organized as a hierarchy that originates from a primitive hematopoietic cell. Nat Med. 1997;3(7):730-737.

2. Dohner $\mathrm{H}$, et al. Acute myeloid leukemia. N EnglJ Med. 2015;373(12):1136-1152.

3. Gentles AJ, et al. Association of a leukemic stem cell gene expression signature with clinical outcomes in acute myeloid leukemia. JAMA. 2010;304(24):2706-2715.

4. Passegue E, et al. Normal and leukemic hematopoiesis: are leukemias a stem cell disorder or a reacquisition of stem cell characteristics? Proc Natl Acad Sci U S A. 2003;100(suppl 1):11842-11849.

5. Lane SW, et al. The leukemic stem cell niche: current concepts and therapeutic opportunities. Blood. 2009;114(6):1150-1157.

6. Greim H, et al. The bone marrow niche, stem cells, and leukemia: impact of drugs, chemicals, and the environment. Ann N Y Acad Sci. 2014;1310:7-31.

7. Dong L, et al. Leukaemogenic effects of Ptpn11 activating mutations in the stem cell microenvironment. Nature. 2016;539(7628):304-308.

8. Duan CW, et al. Leukemia propagating cells rebuild an evolving niche in response to therapy. Cancer Cell. 2014;25(6):778-793.

9. Welner RS, et al. Treatment of chronic myelogenous leukemia by blocking cytokine alterations found in normal stem and progenitor cells. Cancer Cell. 2015;27(5):671-681.

10. Jin L, et al. Targeting of CD44 eradicates human acute myeloid leukemic stem cells. Nat Med. 2006;12(10):1167-1174.

11. Krause DS, et al. Requirement for CD44 in homing and engraftment of BCR-ABL-expressing leukemic stem cells. Nat Med. 2006;12(10):1175-1180.

12. Zheng J, et al. Inhibitory receptors bind ANGPTLs and support blood stem cells and leukaemia development. Nature. 2012;485(7400):656-660.

13. Deng M, et al. LILRB4 signalling in leukaemia cells mediates T cell suppression and tumour infiltration. Nature. 2018;562(7728):605-609.

14. Hao X, et al. Metabolic imaging reveals a unique preference of symmetric cell division and homing of leukemia-initiating cells in an endosteal niche. Cell Metab. 2019;29(4):950-965.

15. Di Virgilio F, Adinolfi E. Extracellular purines, purinergic receptors and tumor growth. Oncogene. 2017;36(3):293-303.

16. Di Virgilio F, et al. Extracellular ATP and P2 purinergic signalling in the tumour microenvironment. Nat Rev Cancer. 2018;18(10):601-618.

17. Idzko M, et al. Nucleotide signalling during inflammation. Nature. 2014;509(7500):310-317.

18. Burnstock G. Physiology and pathophysiology of purinergic neurotransmission. Physiol Rev. 2007;87(2):659-797.

19. Lemoli RM, et al. Extracellular nucleotides are potent stimulators of human hematopoietic stem cells in vitro and in vivo. Blood. 2004;104(6):1662-1670.

20. von Kugelgen I, Wetter A. Molecular pharmacology of P2Y-receptors. Naunyn Schmiedebergs Arch Pharmacol. 2000;362(4-5):310-323.

21. North RA, Surprenant A. Pharmacology of cloned P2X receptors. Annu Rev Pharmacol Toxicol. 2000;40:563-580.

22. Huo JF, Chen XB. P2X4R silence suppresses glioma cell growth through BDNF/TrkB/ ATF4 signaling pathway. J Cell Biochem. 2019;120(4):6322-6329.

23. Jiang $\mathrm{LH}$, et al. ATP-induced $\mathrm{Ca}(2+)$-signalling mechanisms in the regulation of mesenchymal stem cell migration. Cell Mol Life Sci. 2017;74(20):3697-3710.

24. Di Virgilio F, et al. Nucleotide receptors: an emerging family of regulatory molecules in blood cells. Blood. 2001;97(3):587-600.

25. Casati A, et al. Cell-autonomous regulation of hematopoietic stem cell cycling activity by ATP. Cell Death Differ. 2011;18(3):396-404.

26. Rossi L, et al. The extracellular nucleotide UTP is a potent inducer of hematopoietic stem cell migration. Blood. 2007;109(2):533-542.

27. Shabbir M, et al. Purinergic receptor-mediated effects of ATP in high-grade bladder cancer. BJU Int. 2008;101(1):106-112.

28. Communi D, et al. Rapid up-regulation of P2Y messengers during granulocytic differentiation of HL-60 cells. FEBS Lett. 2000;475(1):39-42.

29. van der Weyden L, et al. Extracellular ATP couples to cAMP generation and granulocytic differentiation in human NB4 promyelocytic leukaemia cells. Immunol Cell Biol. 2000;78(5):467-473.

30. Adinolfi E, et al. P2X7 receptor expression in evolutive and indolent forms of chronic B lymphocytic leukemia. Blood. 2002;99(2):706-708.

31. Raffaghello L, et al. The P2X7 receptor sustains the growth of human neuroblastoma cells through a substance P-dependent mechanism. Cancer Res. 2006;66(2):907-914.

32. Adinolfi E, et al. Expression of $\mathrm{P} 2 \mathrm{X} 7$ receptor increases in vivo tumor growth. Cancer Res. 2012;72(12):2957-2969.

33. Bruchard M, et al. Chemotherapy-triggered cathepsin B release in myeloid-derived suppressor cells activates the Nlrp3 inflammasome and promotes tumor growth. Nat Med. 2013;19(1):57-64.
34. Zhang XJ, et al. Expression of P2X7 in human hematopoietic cell lines and leukemia patients. Leuk Res. 2004;28(12):1313-1322.

35 . Wiley JS, et al. A loss-of-function polymorphic mutation in the cytolytic $\mathrm{P} 2 \mathrm{X} 7$ receptor gene and chronic lymphocytic leukaemia: a molecular study. Lancet. 2002;359(9312):1114-1119.

36. Zhang LY, et al. P2X7 polymorphism and chronic lymphocytic leukaemia: lack of correlation with incidence, survival and abnormalities of chromosome 12. Leukemia. 2003;17(11):2097-2100.

37. Feng $\mathrm{W}$, et al. $\mathrm{P} 2 \mathrm{X} 7$ promotes the progression of MLL-AF9 induced acute myeloid leukemia by upregulation of Pbx3 [published online March 12, 2020]. Haematologica. https://doi.org/10.3324/ haematol.2019.243360.

38. Feng W, et al. High level P2X7-mediated signaling impairs function of hematopoietic stem/progenitor cells. Stem Cell Rev Rep. 2016;12(3):305-314.

39. Pelegrin P, Surprenant A. Pannexin-1 mediates large pore formation and interleukin-1beta release by the ATP-gated $\mathrm{P} 2 \mathrm{X} 7$ receptor. EMBO J. 2006;25(21):5071-5082.

40. Karasawa A, et al. The $\mathrm{P} 2 \mathrm{X} 7$ receptor forms a dye-permeable pore independent of its intracellular domain but dependent on membrane lipid composition. Elife. 2017;6:31186.

41. Samways DS, Egan TM. Acidic amino acids impart enhanced $\mathrm{Ca} 2+$ permeability and flux in two members of the ATP-gated P2X receptor family. J Gen Physiol. 2007;129(3):245-256.

42. Amoroso F, et al. The P2X7 receptor is a key modulator of the PI3K/GSK3 $\beta /$ VEGF signaling network: evidence in experimental neuroblastoma. Oncogene. 2015;34(41):5240-5251.

43. Zhang Y, et al. Highly-expressed P2X7 receptor promotes growth and metastasis of human HOS/MNNG osteosarcoma cells via PI3K/Akt/ GSK3 $\beta / \beta$-catenin and mTOR/HIF1 $\alpha /$ VEGF signaling. Int J Cancer. 2019;145(4):1068-1082.

44. Conley JM, et al. Imaging extracellular ATP with a genetically-encoded, ratiometric fluorescent sensor. PLoS One. 2017;12(11):0187481.

45. Lobas MA, et al. A genetically encoded singlewavelength sensor for imaging cytosolic and cell surface ATP. Nat Commun. 2019;10(1):711.

46. Woehrle T, et al. Pannexin-1 hemichannelmediated ATP release together with P2X1 and $\mathrm{P} 2 \mathrm{X} 4$ receptors regulate $\mathrm{T}$-cell activation at the immune synapse. Blood. 2010;116(18):3475-3484.

47. Eltzschig HK, et al. ATP release from activated neutrophils occurs via connexin 43 and modulates adenosine-dependent endothelial cell function. Circ Res. 2006;99(10):1100-1108.

48. Zhang Y, et al. JAM3 maintains leukemia- 
initiating cell self-renewal through LRP5/ AKT/ $\beta$-catenin/CCND1 signaling. JClin Invest. 2018;128(5):1737-1751.

49. Pippel A, et al. Localization of the gate and selectivity filter of the full-length P2X7 receptor. Proc Natl Acad Sci U S A. 2017;114(11):E2156-E2165.

50. Krivtsov AV, et al. Transformation from committed progenitor to leukaemia stem cell initiated by MLL-AF9. Nature. 2006;442(7104):818-822.

51. DeNicola GM, et al. NRF2 regulates serine biosynthesis in non-small cell lung cancer. Nat Genet. 2015;47(12):1475-1481.

52. Possemato R, et al. Functional genomics reveal that the serine synthesis pathway is essential in breast cancer. Nature. 2011;476(7360):346-350.

53. Grol MW, et al. P2 receptor networks regulate signaling duration over a wide dynamic range of ATP concentrations. J Cell Sci. 2013;126(pt 16):3615-3626.

54. Chahwala SB, Cantley LC. Extracellular ATP induces ion fluxes and inhibits growth of Friend erythroleukemia cells. J Biol Chem. 1984;259(22):13717-13722.

55. Patel JJ, et al. Inhibition of vascular smooth muscle cell calcification by ATP analogues. Purinergic
Signal. 2019;15(3):315-326.

56. Schmid R, Evans RJ. ATP-gated P2X receptor channels: molecular insights into functional roles. Annu Rev Physiol. 2019;81:43-62.

57. Jarvis MF, Khakh BS. ATP-gated P2X cation-channels. Neuropharmacology. 2009;56(1):208-215.

58. North RA. Molecular physiology of P2X receptors. Physiol Rev. 2002;82(4):1013-1067.

59. Shabbir M, Burnstock G. Purinergic receptormediated effects of adenosine $5^{\prime}$-triphosphate in urological malignant diseases. Int J Urol. 2009;16(2):143-150.

60. Gu B, et al. Adenosine triphosphate-induced shedding of CD23 and L-selectin (CD62L) from lymphocytes is mediated by the same receptor but different metalloproteases. Blood. 1998;92(3):946-951.

61. Liu Y, et al. Extracellular ATP drives breast cancer cell migration and metastasis via S100A4 production by cancer cells and fibroblasts. Cancer Lett. 2018;430:1-10.

62. Mello Pde A, et al. Adenosine uptake is the major effector of extracellular ATP toxicity in human cervical cancer cells. Mol Biol Cell.
2014;25(19):2905-2918.

63. Cho J, et al. Purinergic P2Y(1)(4) receptor modulates stress-induced hematopoietic stem/progenitor cell senescence. JClin Invest. 2014;124(7):3159-3171.

64. Salvestrini V, et al. Purinergic signaling inhibits human acute myeloblastic leukemia cell proliferation, migration, and engraftment in immunodeficient mice. Blood. 2012;119(1):217-226.

65. Kawata H, et al. Stimulation of cellular senescent processes, including secretory phenotypes and anti-oxidant responses, after androgen deprivation therapy in human prostate cancer. J Steroid Biochem Mol Biol. 2017;165(pt B):219-227.

66. Kang X, et al. The ITIM-containing receptor LAIR1 is essential for acute myeloid leukaemia development. Nat Cell Biol. 2015;17(5):665-677.

67. Chong JH, et al. The hyposensitive N187D P2X7 mutant promotes malignant progression in nude mice. J Biol Chem. 2010;285(46):36179-36187.

68. Adamiak M, et al. Novel evidence that extracellular nucleotides and purinergic signaling induce innate immunity-mediated mobilization of hematopoietic stem/progenitor cells. Leukemia. 2018;32(9):1920-1931. 